\title{
Evidence of powerful relativistic jets in narrow-line Seyfert 1 galaxies
}

\author{
Luigi Foschini ${ }^{* \dagger}$ \\ INAF Osservatorio Astronomico di Brera, Via E. Bianchi 46, 23807, Merate (Italy) \\ E-mail: luigi.foschini@brera.inaf.it
}

In 2008, the Fermi Gamma-ray Space Telescope has revealed - for the first time - high-energy $(\mathrm{E}>100 \mathrm{MeV}) \gamma$ rays from a few Narrow-Line Seyfert 1 Galaxies (NLS1s). Later, in 2009 and 2010, two multifrequency campaigns on one of these sources, PMN J0948+0022 $(z=0.585)$, definitely confirmed the presence, in sources of this type, of a relativistic jet very similar and with comparable power to those in blazars. However, these sources are neither blazars nor radio galaxies, as proven by their optical spectrum and by their very compact radio morphology. Moreover, since NLS1s are generally hosted in spiral galaxies, this casts a significant doubt on the paradigm of the correlation between jets and elliptical host galaxies. These findings pose intriguing challenges to the current knowledge of jet systems and on how these structures are generated. The current status of the researches in this field is reviewed and ongoing work is outlined.

Narrow-Line Seyfert 1 Galaxies and their place in the Universe

April 4-6, 2011

Milano, Italy

\footnotetext{
* Speaker.

$\dagger$ I would like to thank for the interesting, useful, and amusing discussions G. Ghisellini, L. Maraschi, and F. Tavecchio. Thanks also to $\mathrm{S}$. Komossa for the editorial handling of this manuscript.
} 


\section{Introduction}

Narrow-Line Seyfert 1 Galaxies (NLS1s) constitute a class of active galactic nuclei (AGNs) with peculiar characteristics. Before this workshop, I was aware that NLS1s were very interesting sources, but now, after the conclusion of the meeting and having heard several interesting talks, I have understood that NLS1s are even more interesting and special than I could have thought $\left({ }^{1}\right)$.

Richard Pogge in his talk [89] has outlined 25 years of NLS1s studies, from the first interesting notes (e.g. [27, 42]), to the awareness of the discovery of a new class of AGNs [85]. Several authors have pointed out that the observed features at different wavelengths suggest that NLS1s are AGNs with relatively low masses, high accretion rates, and possibly young (e.g. $[14,16,17,50,76$, 88]; see the updates $[18,51,75,87])$. NLS1s generally do not display strong activity at radio frequencies, but a small percent of them $(\sim 7 \%)$ do not follow this rule [65].

The first radio-loud NLS1, PKS 0558-504 ( $\mathrm{z}=0.137)$, was discovered in 1986 [91]. Later, in the early 2000s, a few more sources of this type were discovered [52, 84, 106, 65] and there was already a first (unsuccessful) attempt to detect very high-energy $\gamma$ rays $(E>400 \mathrm{GeV})$ from these sources with the Whipple Čerenkov telescope. More radio-loud NLS1s were found with early surveys both radio- and optically-selected [101, 104]. Although, in some cases, the flat or inverted radio spectrum and high brightness temperature suggested the presence of a relativistic jet [106, 30], in other cases there was no direct indication of beaming [65]. Moreover, these sources had a very compact radio morphology, making it difficult to trace the components searching for superluminal motion [30]. Other indications of a possible presence of a relativistic jet came from optical-to-X-ray studies of variability [37].

The definitive proof of the existence of relativistic jets in NLS1s arrived after the launch of the Fermi Gamma-ray Space Telescope (hereafter Fermi) in 2008 June. Already after a few months of operation, there was the first detection at $\gamma$ rays $(E>100 \mathrm{MeV})$ of a NLS1: it was PMN J0948+0022 $(z=0.5846)[1,38]$. This discovery immediately triggered a multiwavelength (MW) campaign (2009 March-July), which confirmed that the $\gamma$-ray emission was indeed associated with the NLS1 and that the MW behavior was typical of a relativistic jet, like those in blazars [3, 48]. The NLS1 had a peak in the $\gamma$-ray flux on April 1, 2009, with a value of $\sim 4 \times 10^{-7} \mathrm{ph} \mathrm{cm}^{-2} \mathrm{~s}^{-1}$ and then declined, followed by a similar trend at all the wavelengths, while radio emission increased reaching the peak about a couple of months later. In 2010 July, PMN J0948+0022 underwent a much stronger outburst, reaching the power of $\sim 10^{48} \mathrm{erg} / \mathrm{s}$ in the $0.1-100 \mathrm{GeV}$ band and this event was preceded by a significant change in the radio polarization angle, which occurred about one year before [39]. It is worth noting the extreme power at $\gamma$ rays together with the lack of extended radio structures. One basic question, stressed by G. Ghisellini, is indeed: where such great power has gone?

This is just one of the many problems opened by the discovery of high-energy $\gamma$-ray emission from NLS1s, but many others are present. I am not able yet to say what is the effective impact of this discovery in our knowledge of AGNs and relativistic jets. I am still in the phase of searching and collecting informations to better understand the properties of these sources, compare with other known AGNs, and try to infer some useful knowledge. Although there is a handful of $\gamma$-NLS1s,

\footnotetext{
${ }^{1}$ See the workshop proceedings at http://pos.sissa.it/cgi-bin/reader/conf. $\operatorname{cgi}$ ? confid=126.
} 

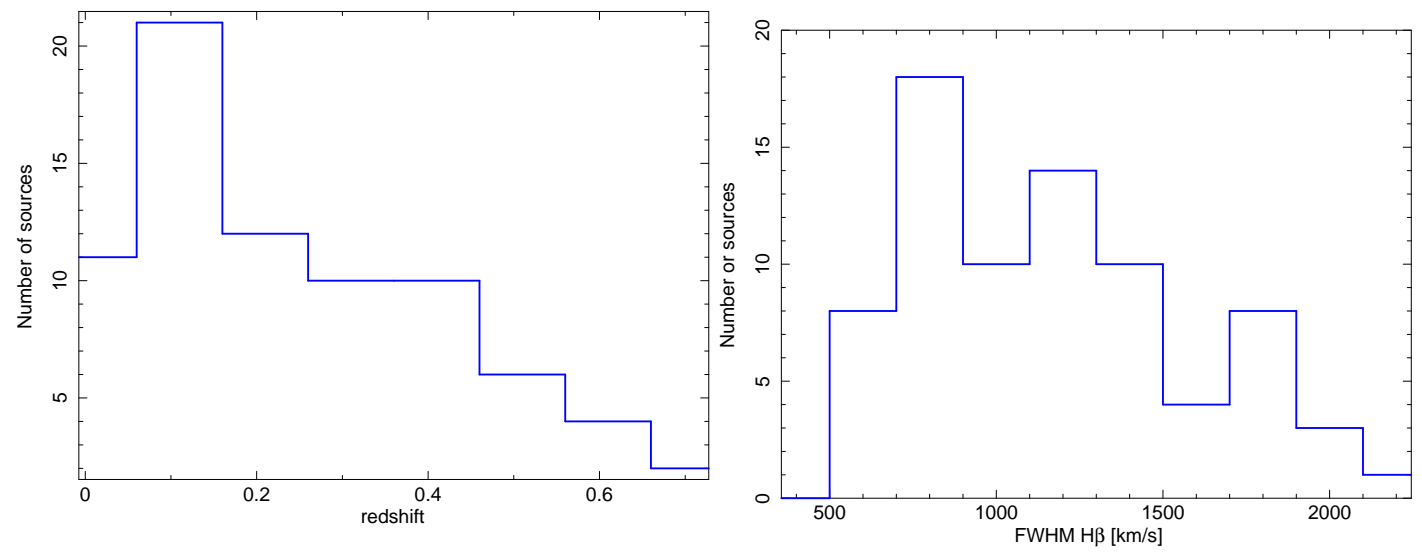

Figure 1: Sample of NLS1s: (left panel) Redshift distribution; (right panel) FWHM H $\beta$ distribution.

in the present work I would like to report about an early study of their MW properties and the comparison with radio-quiet objects and with bright $\gamma$-ray blazars.

\section{Sample selection}

Rather obviously, I performed the early search for high-energy $\gamma$ rays from NLS1s in 2008 by starting with a sample built with the most radio-loud objects. The core of this sample was the list of very radio-loud sources of Yuan et al. [104], which were optically selected from the Sloan Digital Sky Survey (SDSS) and had the radio loudness $R=f_{1.4 \mathrm{GHz}} / f_{440 \mathrm{~nm}}>100$. Some sources with the same $R$ prescription were found in the literature and added to the sample [41, 65, 84, 105, 107]. This list was composed of 29 very radio-loud NLS1s, which resulted in 4 detections at high-energy $\gamma$ rays after one year of Fermi operations [4].

Now, in order to make a more extended work, I have relaxed the prescription on $R$ and added also less radio-loud and even radio-quiet (although not silent) NLS1s from the FIRST Bright Quasars Survey (FBQS) [101]. Therefore, the sample studied in the present work is composed of 76 NLS1s, of which 45 are from the FBQS [101], 23 are from the SDSS [104] and 8 are from literature $[41,65,84,105,107]$ (see Tables A1 and A2 in the Appendix). The radio-quiet $(R<10)$ subsample is composed of 30 sources, while the radio-loud one $(R>20)$ has 39 objects; 7 NLS1s of the present list are radio-intermediate $(10<R<20)$. The distributions of $z$ and FWHM H $\beta$ are displayed in Fig. 1. The measurements of the FWHM H $\beta$ are taken from the available literature ([41, 65, 84, 101, 104, 105, 107]).

I have also considered, as a comparison, a subsample of the bright $\gamma$-ray blazars detected by Fermi during its early months of operations [2]. The subsample of 34 AGNs has been defined on the basis of the availability of Swift optical (B filter) and X-ray data [44, 45]. Radio data were taken from the Faint Images of the Radio Sky at Twenty Centimenters (FIRST), as for the NLS1s (see next Section). It is worth noting that the original selection of these blazars was done on the basis of the high detection significance ( $>10 \sigma,[2])$, so this is not a flux-limited sample. On the other hand, also the sample of NLS1s is not flux limited. 


\section{Multiwavelength Data}

For all the sources in Table A1, I gathered the data from radio to $\gamma$ rays from the publicly available archives and data servers (Table A2). All the data were corrected for the absorption due to the Galactic hydrogen, transformed - when necessary - into flux density at specific frequency (radio: $1.4 \mathrm{GHz}$; optical: $440 \mathrm{~nm}$; X-rays: $1 \mathrm{keV} ; \gamma$ rays: $100 \mathrm{MeV}$ ), $K$-corrected by multiplying the flux density with the factor $(1+z)^{\alpha-1}$, having assumed the following values of $\alpha_{\mathrm{r}}=0, \alpha_{\mathrm{o}}=-0.5$ (see [104]), $\alpha_{\mathrm{X}}=1$, and $\alpha_{\gamma}=1.5$ (see [4]). The corresponding luminosities have been calculated within a $\Lambda$ CDM cosmology with $H_{0}=70 \mathrm{~km} \mathrm{~s}^{-1} \mathrm{Mpc}^{-1}$ and $\Omega_{\Lambda}=0.73$ [64].

\subsection{Radio data}

Radio data were taken from the VLA (Very Large Array) FIRST (Faint Images of the Radio Sky at Twenty Centimenters ${ }^{2}$ ) survey [11], operated at $1.4 \mathrm{GHz}$. Only two sources were in sky regions not covered by the FIRST: PMN J0134-4258 and PKS 0558-504. In both cases, I extended to $1.4 \mathrm{GHz}$ the flux density measured at $4.8 \mathrm{GHz}[103]$ by adopting a flat radio spectrum $\left(\alpha_{\mathrm{r}}=0\right)$.

\subsection{Optical data}

Most of the optical data in the $B$ filter band $(440 \mathrm{~nm})$ were taken from the Sloan Digital Sky Survey $\left(\mathrm{SDSS}^{3}\right.$ ). The ugriz system was converted into the $U B V R I$ one by using the formulae reported in [24]. When the source was not in a region covered by the SDSS, I used the measurements of the US Naval Observatory (USNO) B1 Catalog [81]. For PKS 0558-504, I refer to [82].

The observed magnitudes have been dereddened by using the $A_{\mathrm{V}}$ calculated from the $N_{\mathrm{H}}$ measurements of the Leiden-Argentine-Bonn Survey [62] and the extinction laws by [22].

Most of the sources are quasar-like and, therefore, the host galaxy contribution is negligible. For the lowest redshift sources, I estimated that the host galaxy contributed at maximum by a $10 \%$ in flux, by assuming a spiral host of Sa type ${ }^{4}$.

\subsection{X-ray data}

I have used the ROSAT Bright and Faint Source Catalogues $\left({ }^{5}\right)[98,99]$, which are based on an all-sky survey in the $0.1-2.4 \mathrm{keV}$ energy band. When there is no detection, I calculated a $3 \sigma$ upper limit by using the available exposure at the source position. In a few cases, although there was no detection with ROSAT, the NLS1 was later detected with other satellites (e.g. PKS 1502+036 with Swift, [4]). However, for the sake of homogeneity, I have preferred to keep the upper limit.

The count rates were then dereddened and converted into physical units (c.g.s.) by means of WebP IMMS $\left({ }^{6}\right)$, having frozen the $N_{\mathrm{H}}$ to the Galactic value ([62]) and the photon index of the power-law model to 2 .

\footnotetext{
${ }^{2}$ http://sundog.stsci.edu/index.html

${ }^{3}$ http: //www. sdss.org/

${ }^{4}$ A S0 type would be fainter. See [96].

${ }^{5}$ http://www.xray.mpe.mpg.de/cgi-bin/rosat/rosat-survey

${ }^{6}$ http: //heasarc.gsfc.nasa.gov/Tools/w3pimms.html
} 


\begin{tabular}{|c|c|c|c|c|c|c|c|c|}
\hline Name & RA & Dec & err(dist) & $F_{0.1-100 \mathrm{GeV}}$ & $\Gamma$ & $\mathrm{TS}$ & $|\tau|$ & $\mathrm{S} / \mathrm{N}$ \\
\hline $1 \mathrm{H} 0323+342$ & 51.25 & +34.20 & $0.12(0.07)$ & $6.0 \pm 0.7$ & $2.87 \pm 0.09$ & 164 & $<2.7$ & 4.0 \\
\hline$S B S 0846+513$ & 132.45 & +51.19 & $0.11(0.05)$ & $0.51 \pm 0.15$ & $2.0 \pm 0.1$ & 52 & $12 \pm 8$ & 4.7 \\
\hline PMN J0948+0022 & 147.253 & +0.385 & $0.07(0.02)$ & $13.7 \pm 0.7$ & $2.85 \pm 0.04$ & 1081 & $<0.8$ & 5.4 \\
\hline$F B Q S J 1102+2239$ & 165.70 & +22.63 & $0.37(0.10)$ & $2.0 \pm 0.6$ & $3.1 \pm 0.2$ & 32 & $25 \pm 12$ & 2.9 \\
\hline SDSS J124634.65+023809.0 & 191.83 & +2.53 & $0.47(0.21)$ & $1.7 \pm 0.7$ & $3.1 \pm 0.3$ & 15 & $32 \pm 15$ & 2.1 \\
\hline PKS $1502+036$ & 226.257 & +3.457 & $0.05(0.02)$ & $7.0 \pm 0.6$ & $2.71 \pm 0.07$ & 411 & $1.3 \pm 0.5$ & 6.6 \\
\hline PKS 2004-447 & 302.002 & -44.504 & $0.08(0.07)$ & $1.2 \pm 0.3$ & $2.3 \pm 0.1$ & 44 & $6.2 \pm 1.7$ & 12 \\
\hline
\end{tabular}

Table 1: Summary of Fermi/LAT detections of $\gamma$-NLS1s based on 30 months of data. The new detections are emphasized in italic. The columns "RA" and "Dec" (J2000) indicate the position of the $\gamma$-ray source. The column "err(dist)" is the error at $95 \%$ confidence level [deg] and, between parentheses, there is the distance [deg] of the $\gamma$-ray centroid from the radio coordinates. The three following columns indicate - respectively - the integrated $\gamma$-ray flux in the $0.1-100 \mathrm{GeV}$ energy band $\left[10^{-8} \mathrm{ph} \mathrm{cm}^{-2} \mathrm{~s}^{-1}\right]$, the photon index of the power-law model used to fit the $\gamma$ photons, and the test statistic (TS, see [78] for its definition), where $\sqrt{T S} \sim \sigma$. The two latest columns, $\tau$ and $\mathrm{S} / \mathrm{N}$, display the characteristic time scale [days] of doubling/halving flux and the significance of the flux change $[\sigma]$, respectively.

\section{$3.4 \gamma$-ray data}

I have retrieved all the publicly available data of the Large Area Telescope (LAT, [9]) onboard Fermi included in the period 2008 August 5 00:00 UTC to 2011 February 2 00:00 UTC, i.e. about 30 months of data $\left({ }^{7}\right)$. The analysis was performed by using the LAT Science Tools v. 9.18 .6 and the corresponding background and instrument response files $\left({ }^{8}\right)$. The adopted techniques are quite standard and are described with more details in other papers (e.g. [1, 3, 4]).

First, I have searched for new detections by integrating all the 30 months of data. To take into account the possible contaminating sources inside the $10^{\circ}$ radius of interest centered on the NLS1 under examination, I started modeling the sources in the first Fermi catalog [5]. If, after an inspection of the results of the first analysis run, I found some new contaminating sources, then I added them to the list of modeled sources and repeated the run. The iteration stops when no more newly contaminating sources were found. In addition to the confirmation of the already known $\gamma$-NLS1s [4], I have found three new candidates. The results are summarized in Table 1.

I have also studied the variability at $\gamma$ rays of these sources, by producing light curves in the 0.1-100 GeV energy band with different binnings (1, 2, and 3 days) and taking as good the bins with detections at level $T S>10(\sim 3 \sigma)$. The obtained curves consist of a number of bins spanning from a few points to 87 in the case of PMN J0948+0022, the brightest $\gamma$-NLS1. A few examples of light curves are shown in Fig. 2. It is worth noting the case of SBS $0846+513$, one of the three newly discovered $\gamma$-NLS1s reported in this work: the source was discovered since it became active during the latest months. As I will show also later, this means that the discovery of new $\gamma$-NLS 1 s ultimately depends on the status of activity of the source.

Then, I have analyzed all the lightcurves searching for flux variations greater than $3 \sigma$ and calculated the corresponding time scale $\tau$ for a doubling/halving flux, defined as:

\footnotetext{
${ }^{7}$ http://fermi.gsfc.nasa.gov/cgi-bin/ssc/LAT/LATDataQuery.cgi

${ }^{8}$ http://fermi.gsfc.nasa.gov/ssc/data/analysis/
} 

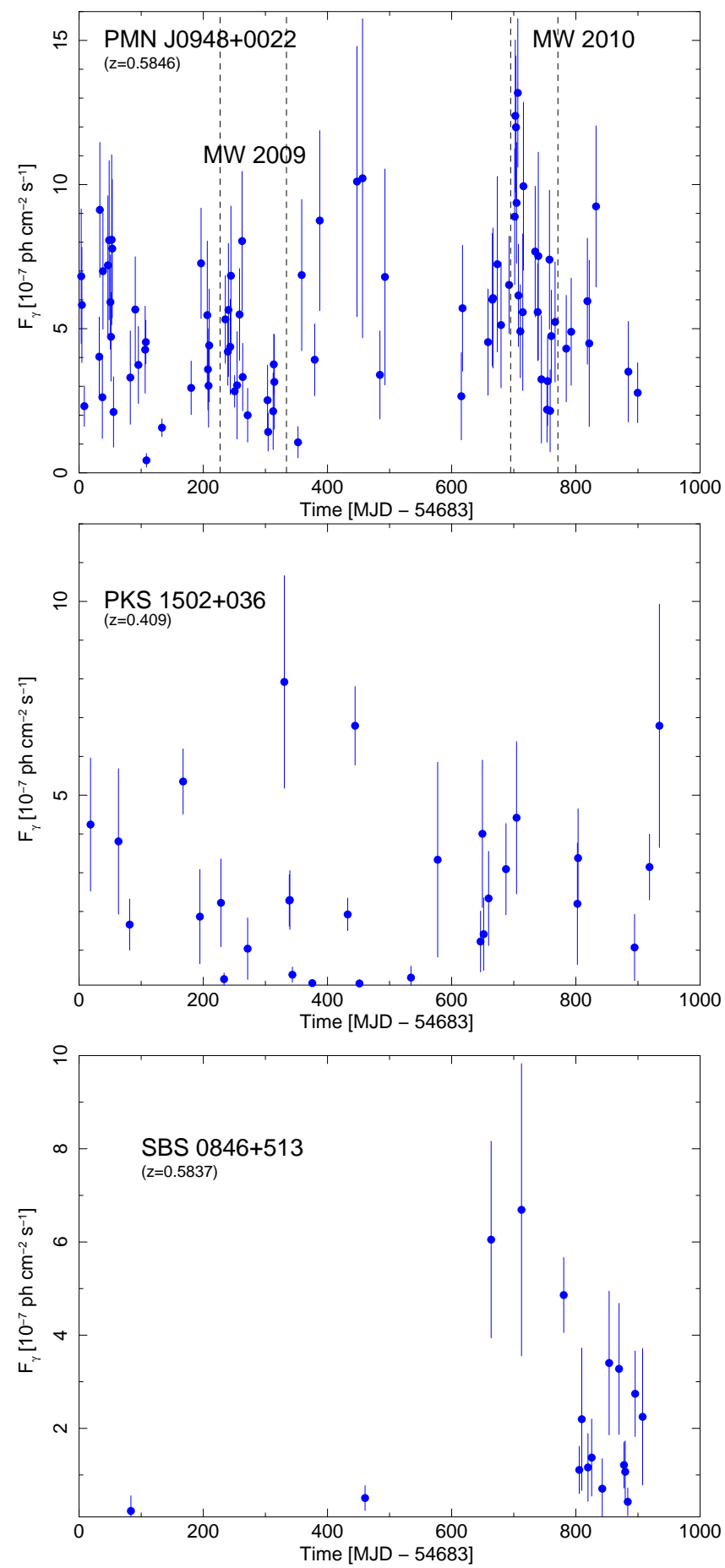

Figure 2: Examples of Fermi/LAT light curves of NLS1s (0.1-100 GeV; 1 day time bin). (top panel) PMN J0948+0022 was the first NLS1 to be detected at $\gamma$ rays $[1,38]$ and was also the target of two MW campaigns in 2009 [3] and 2010 [39], whose periods are indicated with dashed vertical lines; (center panel) PKS $1502+036$ was detected in the first year of operations [4]; (bottom panel) SBS $0846+513$ is a newly detected $\gamma$-NLS1, because it becomes active during the latest months. 

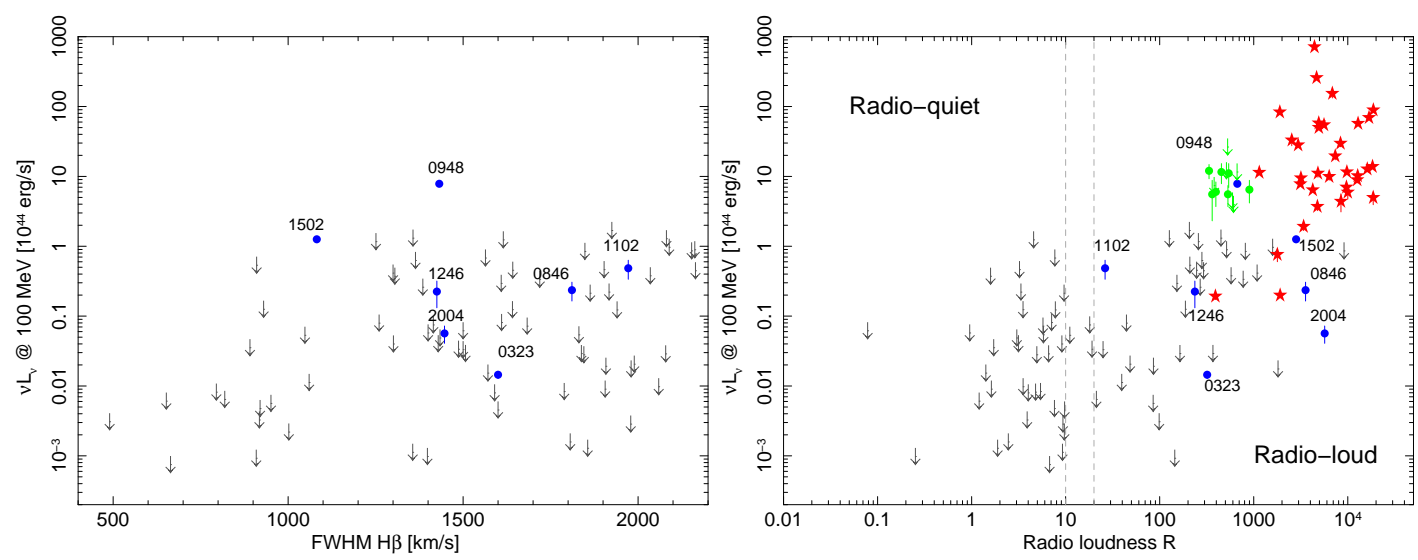

Figure 3: Sample of NLS1s: $\gamma$ rays vs FWHM H $\beta$ (left panel) and radio loudness (right panel). The detections with Fermi/LAT are indicated with blue filled circles. The gray arrows indicate upper limits at $5 \sigma$. The green points refer to the 2009 MW Campaign of PMN J0948+0022 [3]. The red stars are for the bright $\gamma$-ray blazars from the list of $[44,45]$. The two dashed vertical lines represent the separation zone between radio-quiet and radio-loud sources.

$$
\frac{F(t)}{F\left(t_{0}\right)}=2^{-\frac{\left(t-t_{0}\right)}{\tau}}
$$

The results are displayed in Table 1. I have reported also the cases of FBQS J1102+2239 and SDSS J124634.65+023809.0, although the flux variations are not significant. These are also the two faintest $\gamma$-NLS1s, with the poorest statistics for the moment. A better analysis will hopefully be done in the future, if the sources will display some enhanced activity.

\subsection{The case of PMN J0948+0022}

The case of PMN J0948+0022 is worth underlining: there are several observations performed at the time of the discovery of the $\gamma$-ray emission and during the 2009 and 2010 MW Campaigns $[1,3,4,39]$. This offers the possibility to look at the source variations on months time scales and is a reference to understand how much a $\gamma$-NLS1 could change its position along the graphics prepared with MW data described above. To prepare the data, I considered as reference the time of the Swift observations, from which I can measure X-ray and optical fluxes. Fermi information were extracted from a 4-days long subset of LAT data, centered on the Swift observation. The radio fluxes were extrapolated from the closest multifrequency observations by assuming a flat spectrum $\left(\alpha_{\mathrm{r}}=0\right)$.

A caveat should be noted when comparing optical-to- $\gamma$-ray data with radio measurements: indeed, the production of $\gamma$ rays require a compact region, which in turn is also optically thick at radio frequencies [13]. When the blob expands as it moves outward, then it becomes optically thin at radio frequencies, but its $\gamma$-ray production drops. The time lag between the flux peaks at $\gamma$ rays and radio frequencies is of the order of two months for PMN J0948+0022 [3, 39], comparable with the lag observed in blazars (e.g. [66]). For the sake of simplicity, I used the radio measurements closest to the Swift observations to prepare the graphics presented here, but - as I explained - it 


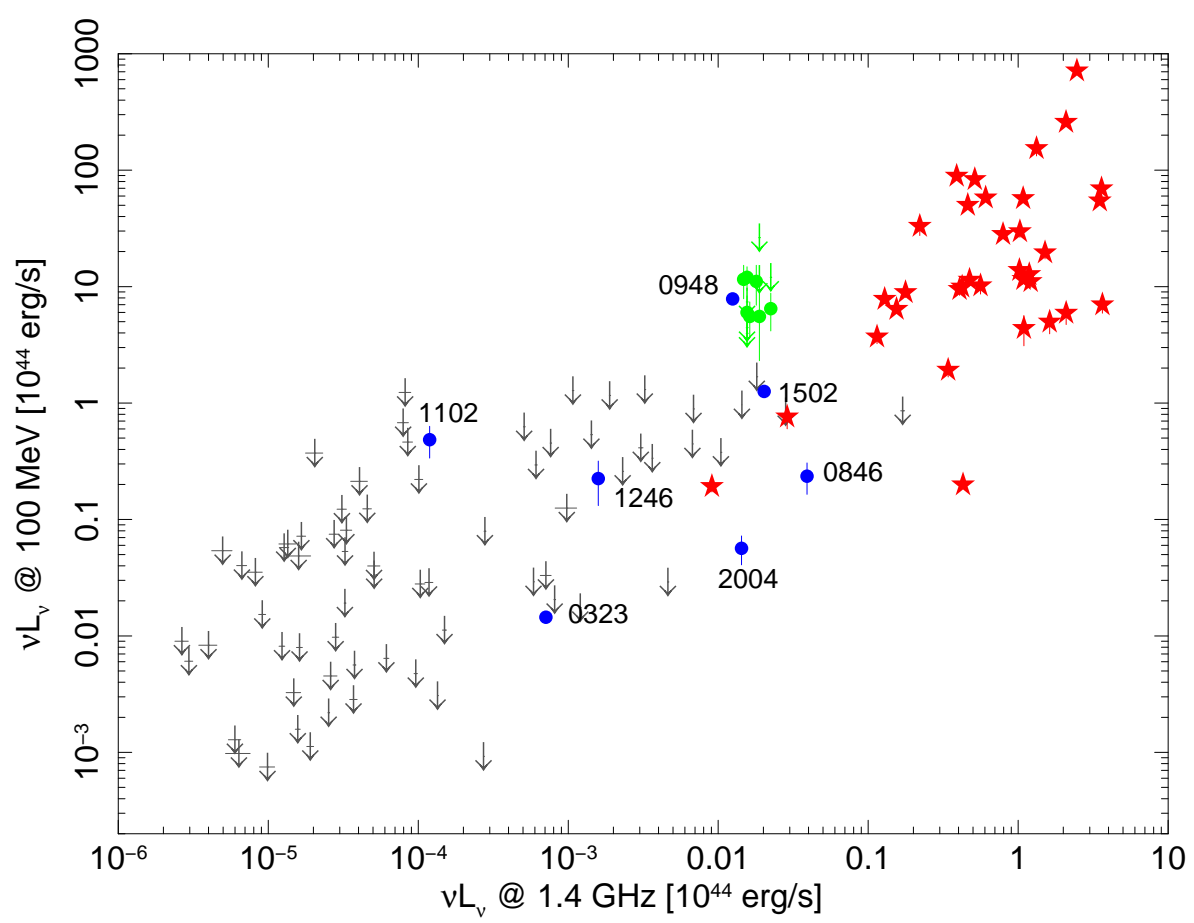

Figure 4: Sample of NLS1s: $\gamma$ rays vs radio. The detections with Fermi/LAT are indicated with blue filled circles. The gray arrows indicate upper limits at $5 \sigma$. The green points refer to the $2009 \mathrm{MW}$ Campaign of PMN J0948+0022 [3]. The red stars are for the bright $\gamma$-ray blazars from the list of [44, 45].

is necessary to take into account their different origin. This caveat is relaxed when dealing with the complete sample of NLS1s, because the observations at different wavelength have been done during specific surveys, which were not linked in time.

\section{Analysis of the sample}

With all the available data described in the previous Section, I prepared the graphics displayed in Fig. 3 and Figs. 4, 5, 6. I searched for correlations of $v L_{v}$ at $100 \mathrm{MeV}$ with its corresponding values at $1.4 \mathrm{GHz}, 440 \mathrm{~nm}$, and $1 \mathrm{keV}$, by using the ASURV Rev. 1.2 software package [67], which makes use of the algorithms described in detail in [35, 60]. Nothing significant was found. When using only the $\gamma$-ray detections, there is something interesting, particularly at optical frequencies (Spearman's $\rho=0.82, P=0.044$ ), but 7 points are not sufficient for a robust correlation. It is necessary to repeat the analysis when more detections will be available. When adding the 34 bright blazars, it is possible to find significant correlations, but it is obviously due to the weight of the latter sample, where correlations are already known (e.g. [8]).

No clear trend is visible in the FWHM H $\beta$ of the $\gamma$-NLS1s with respect to the other NLS1s of the sample, as well as in the radio loudness, except for the fact that all the $\gamma$-NLS1s are radio-loud (Fig. 3). The least radio-loud $\gamma$-NLS1 is FBQS J1102+2239, with $R=32$. The source is rather unknown, but we could expect some degree of variability in the radio loudness, as shown by the changes of the points of PMN J0948+0022 (maximum variation by a factor 2.6). 


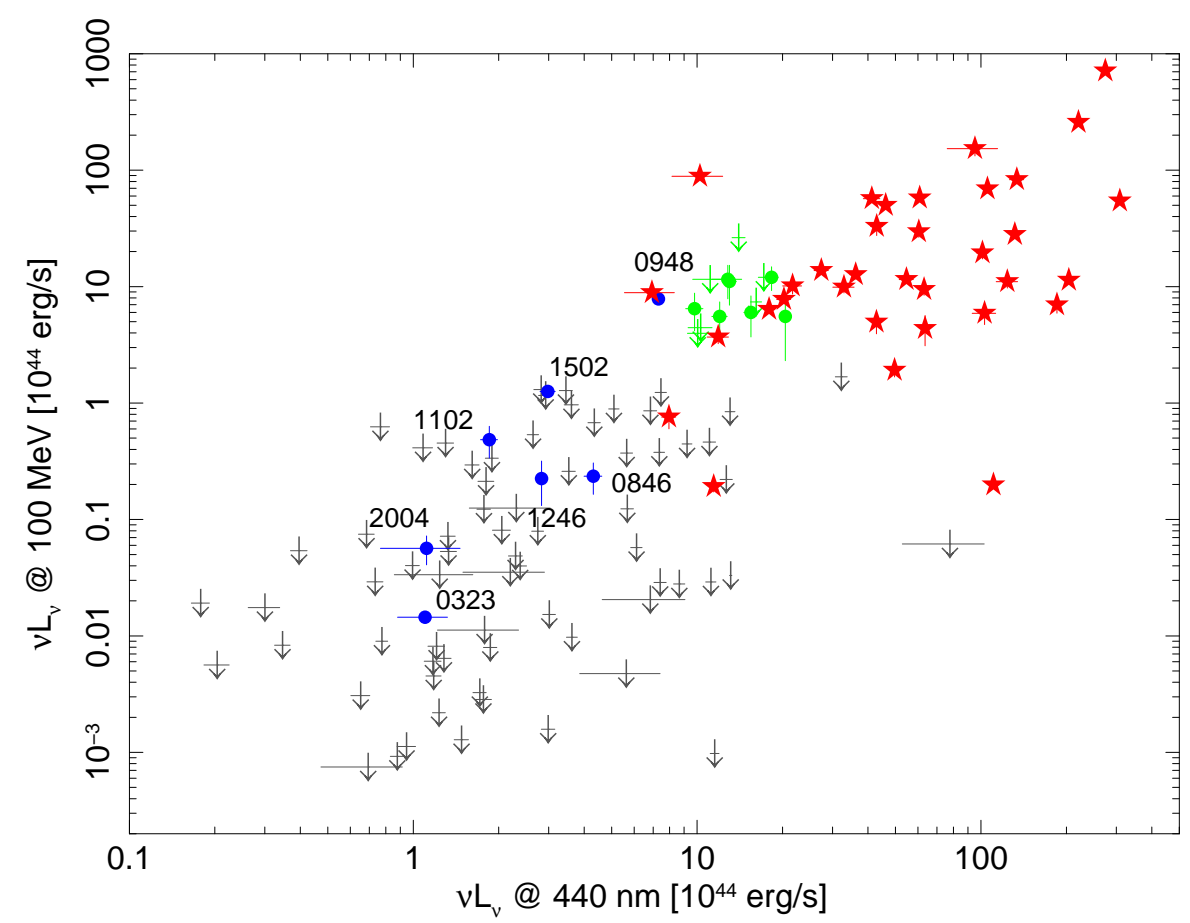

Figure 5: Sample of NLS1s: $\gamma$ rays vs optical. The detections with Fermi/LAT are indicated with blue filled circles. The gray arrows indicate upper limits at $5 \sigma$. The green points refer to the $2009 \mathrm{MW}$ Campaign of PMN J0948+0022 [3]. The red stars are for the bright $\gamma$-ray blazars from the list of $[44,45]$.

\begin{tabular}{lcccccccc}
\hline Quantities & \multicolumn{3}{c}{ NLS1s } & \multicolumn{4}{c}{ Blazars + NLS1s } \\
\hline & \multicolumn{2}{c}{ Kendall } & \multicolumn{2}{c}{ Spearman } & \multicolumn{2}{c}{ Kendall } & \multicolumn{2}{c}{ Spearman } \\
\hline & $Z$ & $P$ & $\rho$ & $P$ & $Z$ & $P$ & $\rho$ & $P$ \\
\hline$L_{100 \mathrm{MeV} \text { vs } L_{1.4 \mathrm{GHz}}}$ & 3.5 & $5 \times 10^{-4}$ & 0.59 & $<10^{-4}$ & 9.2 & $<10^{-4}$ & 0.86 & $<10^{-4}$ \\
$L_{100 \mathrm{MeV} \text { vs } L_{440 \mathrm{~nm}}}$ & 1.2 & 0.23 & 0.30 & $9 \times 10^{-3}$ & 8.2 & $<10^{-4}$ & 0.75 & $<10^{-4}$ \\
$L_{100 \mathrm{MeV} \text { vs } L_{1 \mathrm{keV}}}$ & 1.9 & 0.052 & 0.27 & 0.02 & 9.1 & $<10^{-4}$ & 0.76 & $<10^{-4}$ \\
$L_{100 \mathrm{MeV} \text { vs } R L} L$ & 3.4 & $8 \times 10^{-4}$ & 0.49 & $<10^{-4}$ & 8.5 & $<10^{-4}$ & 0.81 & $<10^{-4}$ \\
\hline
\end{tabular}

Table 2: Summary of the correlation analysis. The number of points for the NLS1s sample is 76, with 69 upper limits at $\gamma$ rays and 28 upper limits at X-rays. The number of points of the Blazars + NLS1s sample is 110 , with the same number of upper limits. $P$ is the chance probability.

Among the faintest radio sources, $1 \mathrm{H} 0323+342$ is the closest $\gamma$-NLS1, so in this case the low radio luminosity is compensated by the proximity of the source. Indeed, its flux density is not negligible ( $\sim 600 \mathrm{mJy}$; see Table A1). The two other sources, FBQS J1102+2239 and SDSS J124634.65+023809.0, are farther, with $z=0.455$ and 0.362 , respectively. Their fluxes are weak: $\sim 2$ and $\sim 38 \mathrm{mJy}$, respectively (see Table A1), so there is some doubt that the $\gamma$-ray detection could be due to chance coincidence $\left({ }^{9}\right)$. These sources are anyway radio-loud, clearly because they are much more faint at optical frequencies. It is worth noting a bright Fermi blazar close to the position of SDSS J124634.65+023809.0 in the $\gamma$-ray vs radio loudness graphic (Fig. 3, right panel), which is $\mathrm{OX}+169(z=0.21)$, although in term of radio power the difference between the

\footnotetext{
${ }^{9}$ SDSS J124634.65+023809.0 is also the weakest detection at $\gamma$-rays with $T S=15$, i.e. $\sim 4 \sigma$. See Table 1.
} 


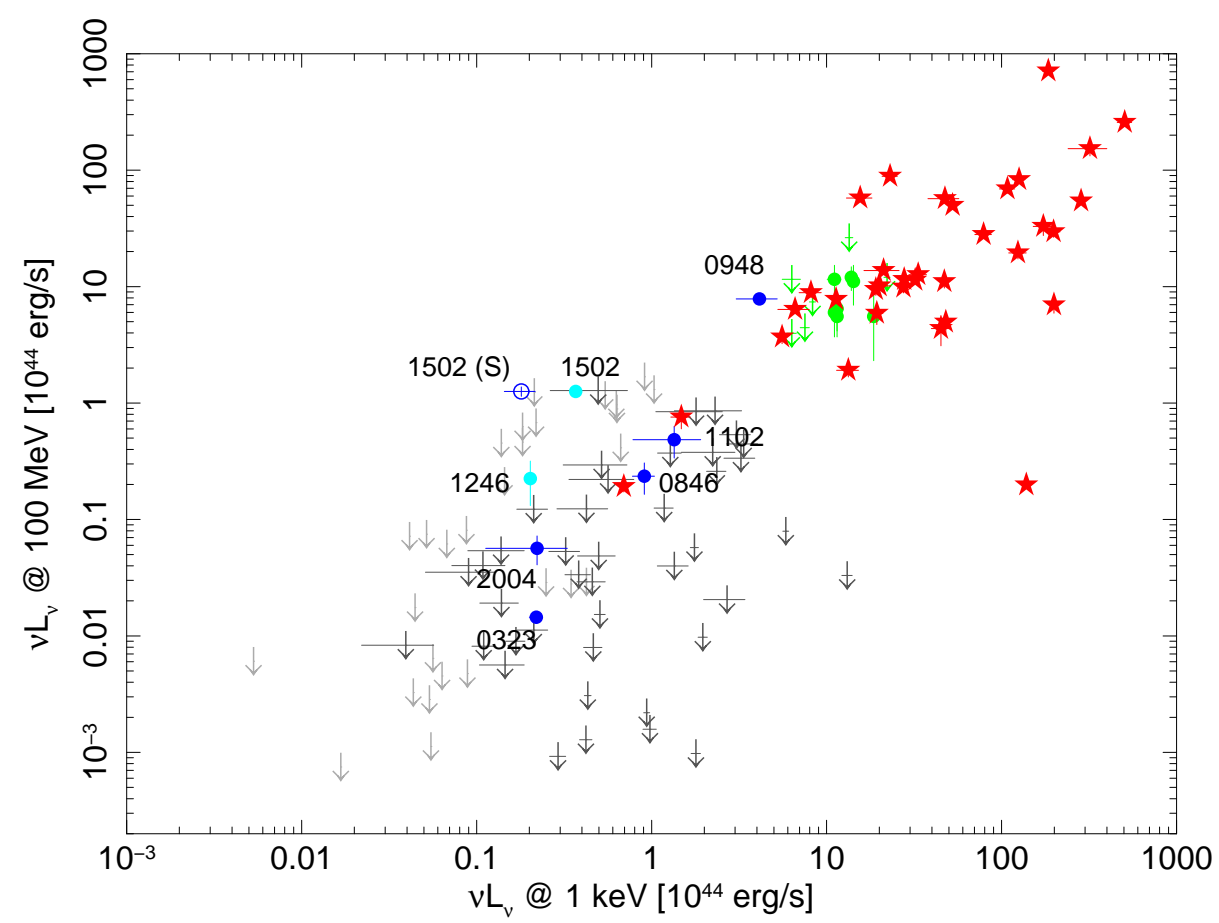

Figure 6: Sample of NLS1s: $\gamma$ rays vs X-rays. The detections with Fermi/LAT are indicated with blue filled circles. The gray arrows indicate upper limits at $5 \sigma$. The green points refer to the $2009 \mathrm{MW}$ Campaign of PMN J0948+0022 [3]. The red stars are for the bright $\gamma$-ray blazars from the list of [44, 45]. If the source is not detected by ROSAT, then it is reported the $3 \sigma$ upper limit. The sources not detected both by ROSAT and Fermi are indicated with light gray arrows, while the sources detected by Fermi, but not by ROSAT are in light blue. In the case of PKS 1502+036, the detection with Swift [4] is indicated with an open blue circle.

two is about one order of magnitude (see Fig. 4). There are other blazars with such low radio fluxes, at mJy level [74], some of them detected at high-energy $\gamma$ rays [5]. Therefore, although the radio fluxes of the two NLS1s are low, it is not unlikely that they can generate high-energy photons, as it happens for blazars with comparable fluxes.

I tried to estimate the brightness temperature, by using both the approaches based on the spectral characteristics [90] and the variability at radio frequencies [100]. In the latter case, there are two measurements done by the FIRST [11] and by the NRAO VLA Sky Survey (NVSS, [25]), which were done with a few years of time separation. In the case of FBQS J1102+2239, there is a change of about $67 \%$ between the measurement performed during the NVSS (1993 Dec 6, 2.7 $\pm 0.5 \mathrm{mJy}$ ) and FIRST (1996 Jan, 1.8 $\pm 0.1 \mathrm{mJy}$ ), which results in an estimation of the brightness temperature of $\sim 10^{11} \mathrm{~K}$. Instead, SDSS J124634.65+023809.0 does not show evident indications of variability, although the two measurements were done on much larger timescale (NVSS, $36 \pm 1 \mathrm{mJy}, 1995$ Feb 27; FIRST, $38 \pm 1 \mathrm{mJy}, 1998$ July). The brightness temperature is then $\sim 5 \times 10^{10} \mathrm{~K}$. The estimation following [90] and assuming a rather flat spectral index of $\alpha=0.1$, gives a temperature of $\sim 7 \times 10^{10} \mathrm{~K}$ in both cases, which is comparable with the values obtained with the variability. This value obviously increases to exceed $10^{11} \mathrm{~K}$ if the spectral index becomes inverted. These temperatures are not outstandingly indicative of beamed non-thermal emission, but there is any- 
way some room for hope. Further observations are needed to better understand these sources and L. Fuhrmann, E. Angelakis et al. are planning multifrequency pointings with the Effelsberg radio telescope.

Figs. 5 and 6 display the $\gamma$ rays vs optical and X-ray data. As previously stated, there is some hint of correlation between optical and $\gamma$ rays when only the $\gamma$-NLS1s are considered. This can be explained having in mind the behavior observed in the 2009 MW Campaign of PMN J0948+0022 [3], where a change in the optical emission due to the synchrotron emission was observed. This is confirmed by the recent polarization measurements in the $V$ filter performed by the Kanata telescope, which found a $19 \%$ of polarization, comparable with the greatest value of the brightest blazars [58]. Also the optical emission of $1 \mathrm{H} 0323+342$ is polarized, but with a lower degree $(0.7-0.8 \%)$, although still in the range of other blazars $[58]\left({ }^{10}\right)$.

The fact that the $\gamma$-NLS1s are not clustered around some extreme values of $L_{1.4 \mathrm{GHz}}, L_{440 \mathrm{~nm}}$, $L_{1 \mathrm{keV}}$, and radio loudness makes it clear that the measurements of high-energy $\gamma$ rays is still strongly dependent on the activity of the source. Indeed, PMN J0948+0022 is the most active $\gamma$-NLS1 of these years and SBS $0846+513$ was detected only recently, because of an increase of activity. This means that more discoveries are expected from a continuous monitoring of NLS1s.

\section{Host galaxy}

It is well-known the paradigm that associates the presence of powerful relativistic jets with elliptical host galaxies, while radio-quiet AGNs are hosted by spirals or even ellipticals when at high redshift (e.g. $[10,63,68,93])$. Some researchers suggested that this could be due to a requirement of great masses in the jet formation, where AGNs with jets need of $M>10^{9} M_{\odot}$ and those without jets have $M<3 \times 10^{8} M_{\odot}$ ([68], but see also [40]). The former are common in ellipticals, while the latter are present in spirals.

The jet-elliptical paradigm was also the basis for elaborating a blazar evolution sequence, where highly-accreting and distant quasars evolve into lowly-accreting nearby BL Lac objects as the fueling of the central supermassive black hole is going to finish [15, 23, 73].

Although Ho \& Peng [56] have demonstrated that many Seyferts (hosted by spirals) become radio-loud once the nuclear luminosity is correctly measured, the counterexamples to this paradigm were extremely rare (e.g. [19, 20]; see the next Section). Today, it is clear that almost no Seyfert is really radio-silent, but there is more or less frequently some radio emission that can be interpreted as the basis of a jet or an outflow [55, 47]. However, there is no general consensus on a specific explanation. Most important, none of these source was never detected at high-energy $\gamma$ rays: the recent detection with Fermi of GeV $\gamma$ rays from the Seyfert 2s NGC 1068 and NGC 4945 can be explained with the emission from the starburst component and the proximity of the two sources $[71]\left({ }^{11}\right)$.

Some more cases of interesting flat spectrum and high brightness temperature radio emission were found in NLS1s (generally hosted by spirals), as reminded in the Introduction, and indeed these hints of powerful relativistic jets were then confirmed by the detection of $\mathrm{GeV}$ photons from

\footnotetext{
${ }^{10}$ In [58], the two $\gamma$-NLS1s are erroneously classified as FSRQs.

${ }^{11}$ Although, NGC 1068 might have also some contribution from a jet [71], but more observations are needed to better understand this source.
} 


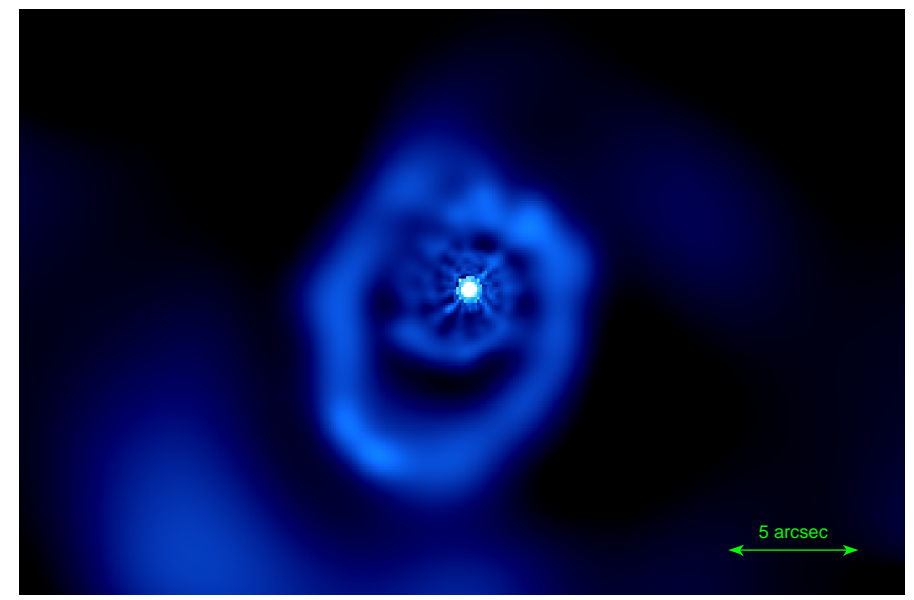

Figure 7: Hubble Space Telescope/WFPC2 image of $1 \mathrm{H} 0323+342(z=0.061)$ with the filter F702W $(690 \mathrm{~nm})$. Two observations $200 \mathrm{~s}$ long were integrated and then adaptively smoothed [31] to emphasize the low brightness structures.

a handful of NLS1s. Among the $\gamma$-NLS1s, only one can be imaged with sufficient resolution to understand its host galaxy: it is $1 \mathrm{H} 0323+342(z=0.061)$, which was observed by the Hubble Space Telescope. These observations have shown a spiral arm [107], although other observations from the Nordic Optical Telescope (NOT) seem to suggest a circum-nuclear starburst ring, probably the trail of a recent merger [7]. The two options are not in contrast, because these starburst rings are common among NLS1s [29] and the star forming activity is stronger in NLS1s than in other Seyferts [92]. I have downloaded the $H S T$ data and performed some image analysis, which resulted in an emphasis of the low brightness structures. As shown in Fig. 7, the spiral structure is now more evident.

Although no high-resolution observations are available for the remaining $\gamma$-NLS1s, it is likely that they are hosted by spirals, because this type of galaxy hosts almost all the low redshift NLS1s $[26,29]$. Moreover, the presence of pseudobulges - which are slowly built from spiral discs - is in favor of the hypothesis that NLS1s are young active nuclei still growing by secular evolution $\left({ }^{12}\right)$ $[77,83]$. The intense starburst activity can be the result of a high accretion rate, supported by the large presence of bars, which in turn produce shocks in the interstellar medium and hence trigger the star formation. The presence of powerful relativistic jets might be a consequence of this young environment, because the jet can enhance the accretion, by draining angular momentum [61]. So, $\gamma$-NLS1s might be the low-redshift analog of high-redshift young quasars. The latter evolve into BL Lac Objects at low- $z$. What will be the fate of $\gamma$-NLS1s? Is it still to be written?

\section{Search for the parent population}

The presence of a population of AGNs with beamed jets poses the question of the parent population, i.e. those sources of the same type but viewed at random orientations. The number of the parent population is $\sim 2 \Gamma^{2}$ times the number of beamed sources. Therefore, if we know 7

\footnotetext{
${ }^{12}$ Indeed, no NLS1 has been found a $z \gtrsim 0.8$.
} 


\begin{tabular}{|c|c|c|c|c|c|c|c|c|c|}
\hline Name & $\overline{\mathrm{RA}}$ & $\overline{\text { Dec }}$ & $\mathrm{Z}$ & Host & Ref. & $F_{0.1-100 \mathrm{GeV}}$ & $\bar{\Gamma}$ & $\mathrm{TS}$ & err(dist) \\
\hline III Zw 2 & 2.6283 & +10.9703 & 0.090 & Sab? & {$[19,20]$} & $<2.0$ & & & \\
\hline NGC 612 & 23.4906 & -36.4932 & 0.030 & $\mathrm{Sa}$ & [32] & $<0.14$ & & & \\
\hline PMN J0315-1906 & 48.9671 & -19.1123 & 0.067 & S0ab? & {$[69,70]$} & $<0.08$ & & & \\
\hline PKS 0336-177 & 54.8070 & -17.6002 & 0.065 & So & [72] & $0.25 \pm 0.14$ & $1.7 \pm 0.2$ & 80 & $0.045(0.026)$ \\
\hline $3 C 120$ & 68.2962 & +5.3543 & 0.033 & So & [80] & $<2.2$ & & & \\
\hline B2 $0722+30$ & 111.4057 & +29.9541 & 0.019 & So & [33] & $<0.12$ & & & \\
\hline $3 C 277.1$ & 193.1098 & +56.5721 & 0.320 & S0? & {$[53,54]$} & $<0.03$ & & & \\
\hline PG $1309+355$ & 198.0740 & +35.2559 & 0.183 & Sab & {$[53,54]$} & $<2.3$ & & & \\
\hline PKS $1413+135$ & 213.9951 & +13.3399 & 0.247 & $\mathrm{Sa}$ & [86] & $4.1 \pm 0.6$ & $3.0 \pm 0.1$ & 119 & $0.075(0.051)$ \\
\hline
\end{tabular}

Table 3: Sample of radio galaxies in spiral hosts. The coordinates are at the epoch J2000. The $\gamma$-ray flux is in units of $\left[10^{-8} \mathrm{ph} \mathrm{cm}^{-2} \mathrm{~s}^{-1}\right]$; upper limits are at $5 \sigma$ confidence level. The last column, indicating the $95 \%$ error circle of the LAT source and the distance from the radio position, is in [deg]. PKS 0336-177 is also present in the first LAT catalog [5]. 3C 120 is not present in the first LAT catalog [5], although a detection is reported in [6].

$\gamma$-NLS1s, this means that there should be $\sim 1400$ unbeamed parent sources, having assumed the common value of $\Gamma=10$. The parent population of blazars is that of the radio galaxies, but what is the parent population of $\gamma$-NLS1s?

The only known NLS1 displaying extended radio structures on kpc scale is PKS 0558-504, with a $\sim 46 \mathrm{kpc}$ bipolar jet detected at $4.8 \mathrm{GHz}$ [49]. The extreme compactness and lack of extended structures of the $\gamma$-NLS1s at radio frequencies [30, 104, 39, 48] might suggest that when observed at large angles, these sources simply becomes radio-quiet, because there is negligible unbeamed radio emission. In this case, the parent population of $\gamma$-NLS1s might simply be that of radio-quiet NLS1s.

There could be another possibility. If the broad-line region (BLR) of NLS1s is disc-like and we are observing it pole-on, then there is no motion along the viewing angle, the Doppler broadening is negligible, and the profiles of the emission lines of the BLR are narrower than usual [28]. This has been observed also when analyzing large samples of radio-loud AGNs and explained with a disc-like BLR viewed at small angles [79]. In the case of NLS1s, this means that when observed at large angles, they become the usual broad-line Seyferts. Therefore, the parent population should be searched among the broad-line radio galaxies hosted by spirals, which are not very common [97]. Only recently, Inskip et al. performed a near-IR morphological study on the 2 Jy sample of radio-loud AGNs and found that $\sim 12 \%$ of the sources are hosted by disc galaxies [59]. In the past, the examples of radio-loud AGNs in spirals were just a few (e.g. [53, 54]). Others can be found in the literature, but they were a handful (see Table 3). It is worth noting that often these sources are hosted by $\mathrm{S} 0$ galaxies and, therefore, there could be the risk of misclassification: a faint elliptical galaxy can be erroneously classified as a bright S0, and vice versa.

Therefore, the parent population of the $\gamma$-NLS1s is still basically unknown.

\section{Implications on the classification of AGNs}

Before the discovery of $\gamma$-NLS1s, the AGNs with relativistic jets viewed at small angles were called blazars. Blazars were divided into FSRQ and BL Lac Objects, depending on the equivalent width of their emission lines ( $\gtrless 5 \AA$; e.g. [95]), which in turn translates in an indication of the 


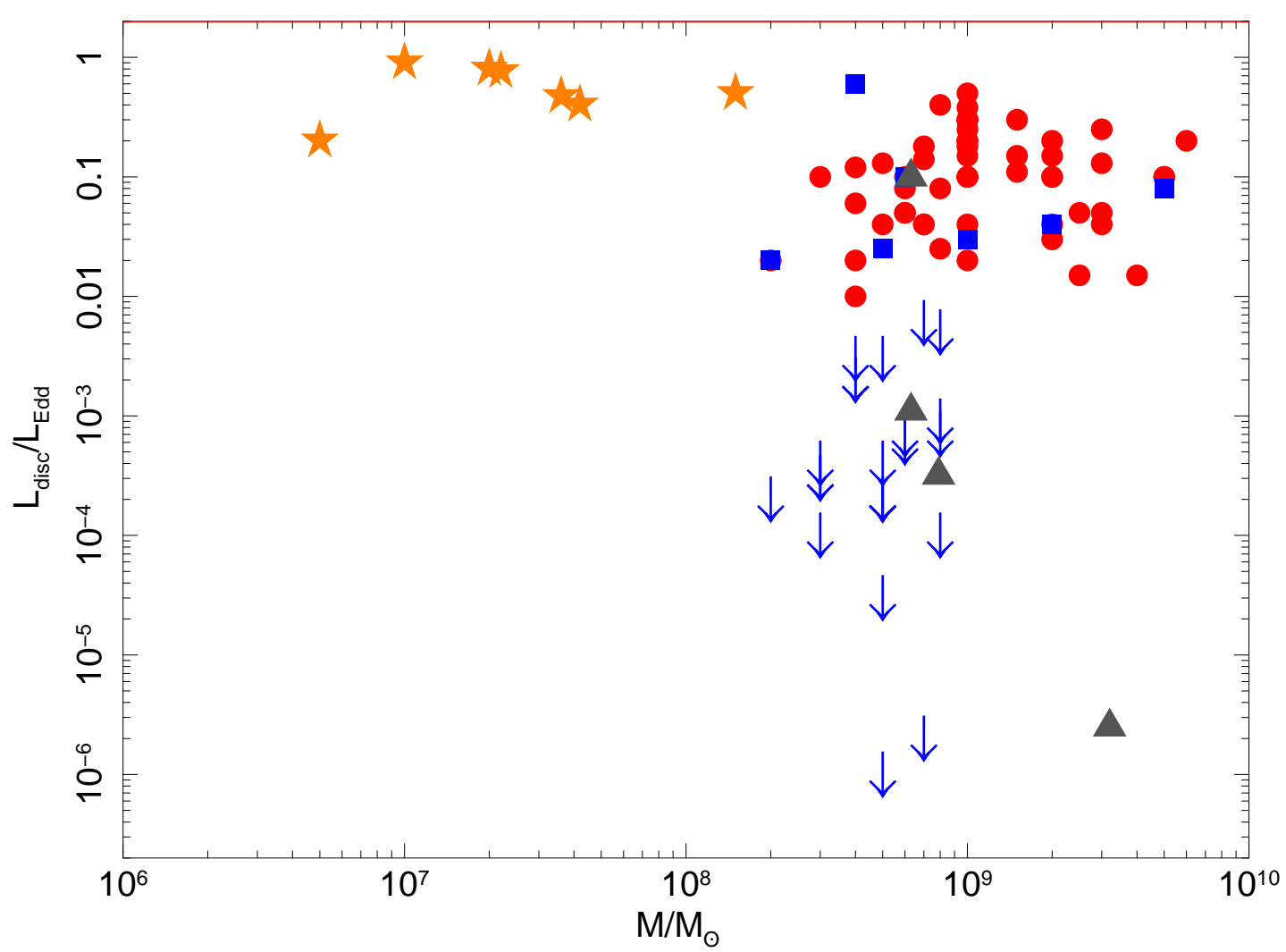

Figure 8: AGNs with relativistic jets in terms of mass and accretion power (in Eddington units). FSRQ are indicated with red circles and BL Lac Objects are represented with blue squares or arrows, in the case it was not possible to estimate the accretion power and there is only an upper limit (data from [45]). Some radio galaxies detected at $\gamma$ rays [6] are indicated with gray triangles (data from [93]). Orange stars are for $\gamma$-NLS1s (data for the first four are from [4], while the three newly detected are from the present work).

accretion power $\left({ }^{13}\right)$. The same type of host suggested an evolutionary link between the two types of sources. Therefore, the term blazar indicates an AGN with a relativistic jet viewed at small angles, hosted in an elliptical galaxy, with an accretion disc and a BLR more or less developed, depending on the luminosity of the disc. The quasars have high accretion $\left(\sim 0.1 L_{\text {Edd }}\right)$ and fully developed BLR, while BL Lacs have low accretion $\left(<10^{-3} L_{\text {Edd }}\right)$ and weak BLR (e.g. [45]). The FWHMs of the lines were always $>2000 \mathrm{~km} / \mathrm{s}[36,102]$. The masses of the central black hole are in the range of $10^{8-10} M_{\odot}$ (e.g. [45]). They were born as FSRQ and evolve into BL Lac Objects, as the fuel is ending $[15,23,73]$. Therefore, it is the same type of source (blazar) and the terms FSRQ and BL Lac Object refer to specific evolutionary stages, where the nearby environment can determine a more or less efficient cooling of the relativistic electrons of the jet [43].

Some researchers use the term blazar to indicate only the emission from the relativistic jet viewed at small angles, because it overwhelms almost all the other components of the AGN. Sometimes, it is possible to read terms like "quasar-hosted blazars" (e.g. [94]). However, I think that

\footnotetext{
${ }^{13}$ Recently, Ghisellini et al. [46] proposed to revise this classification on the basis of the line luminosities in Eddington units. In this case, the threshold is at $L_{\mathrm{BLR}} / L_{\mathrm{Edd}} \sim 5 \times 10^{-4}$.
} 


\begin{tabular}{crr}
\hline \multicolumn{2}{c}{ Beamed } & \multicolumn{2}{c}{ Unbeamed (parent population) } \\
\hline Blazars: & Radio Galaxies: \\
& - FSRQ & - High-Excitation RG \\
- BL Lacs & - Low-Excitation RG \\
\hline$\gamma$-NLS1s & RGs in spirals or misaligned NLS1s? \\
\hline
\end{tabular}

Table 4: Tentative classification of AGNs with relativistic jets. For the definition of High- and LowExcitation RG see, for example, [21].

this terminology is misleading for a couple of reasons: it is rather evident that several important informations are lost, specifically on the AGN components and hosts, and therefore it is necessary to add other words to recover this information, as in the above example. Moreover, there could be confusion with relativistic jets in Galactic compact objects: if the term blazar should indicate only the emission from a jet viewed at small angles, then also microquasars should be simply named blazars.

Now, with the arrival of $\gamma$-NLS1s, the problems are increasing. Indeed, if we continue naming blazars any AGN with a relativistic jet viewed at small angles, we should call in this way also the $\gamma$-NLS1s, thus losing a lot of informations and questions. $\gamma$-NLS1s are different from blazars in a lot of key aspects: smaller masses $\left(10^{6-8} M_{\odot}\right)$, greater accretion rates $\left(>>0.1 L_{\text {Edd }}\right.$, up to the Eddington value), smaller FWHMs of the BLR lines ( $<2000 \mathrm{~km} / \mathrm{s})$, different hosts (spiral). Surely the jet in $\gamma$-NLS1s is very similar to the jets in blazars (see Fig. 1 in [38]), but everything else is different. Indeed, as we plot a graphic with accretion power vs central mass, then the difference between $\gamma$-NLS1s, blazars and radio galaxies is immediately evident (see Fig. 8).

Therefore, I think it is better to keep the name blazars for FSRQ plus BL Lacs, so that this term refers to a global set of properties of the whole AGN and its host, rather than only of the jet. To summarize, we could then divide the AGNs with relativistic jets according to the Table 4.

The evidence of powerful relativistic jets in NLS1s confirmed that these structures are rather ubiquitous in the Universe and that the jet-elliptical paradigm was an observational bias. It is worth reminding the exchange of words by R. Blandford and G. Burbidge in the discussion after Blandford's seminal talk at the "Pittsburgh Conference on BL Lac Objects" in 1978 [12]:

G. Burbidge: "Roger, is your model for AO $0235+164$ an elliptical galaxy with a black hole at the center?"

R. Blandford: "I would say that it is either a galaxy or a proto-galaxy. As the continuum emission is proposed to originate in the central $10 \mathrm{pc}$, I don't think the nature of the surrounding object is particularly relevant to the model." [the emphasis is mine].

\section{Prospects}

I prefer to write something on the future, rather than to write a conclusion section. Indeed, the discovery of $\gamma$-NLS1s opens many more questions rather than to solve any single doubt. Something has been done, but much more has still to be done. I have no answers, but a lot of questions. In my opinion, the key points in this research field are: 
- The first point is rather obvious: improve the sample of $\gamma$-NLS1s! As the detection depends on the activity of the source, the monitoring of the sky with Fermi guarantees an open space for new discoveries. With more sources, it will be possible to search for correlations and better assess the statistical properties of $\gamma$-NLS1s.

- Study the time-resolved MW properties of $\gamma$-NLS1s: a few detailed cases study can often offer many more and deeper informations on the nature of certain cosmic sources than a statistical study on a wide sample.

- Study the host galaxy: to date, only one $\gamma$-NLS1 is known to be hosted in a spiral galaxy $(1 \mathrm{H} 0323+342)$, while for the others we can infer the same host type. Obviously, one thing is the inference, another thing is the direct observation.

- Search for the parent population: in this case, it is not yet clear if we have to search for other NLS1s with kpc structures, like PKS 0558-504, or if the answer is among the broad-line radio galaxies in spiral hosts.

\section{References}

[1] A. A. Abdo et al. (Fermi/LAT Collaboration): Fermi Large Area Telescope discovery of gamma-ray emission from a relativistic jet in the narrow-line quasar PMN J0948+0022, ApJ 699 (2009) 976.

[2] A. A. Abdo et al. (Fermi/LAT Collaboration): Bright active galactic nuclei source list from the first three months of the Fermi Large Area Telescope all-sky survey, ApJ 700, (2009), 597.

[3] A. A. Abdo et al. (Fermi/LAT Collaboration): Multiwavelength monitoring of the enigmatic narrow-line Seyfert 1 PMN J0948+0022 in 2009 March-July, ApJ 707 (2009) 727.

[4] A. A. Abdo et al. (Fermi/LAT Collaboration): Radio-loud narrow-line Seyfert 1 as a new class of gamma-ray active galactic nuclei, ApJ 707 (2009) L142.

[5] A. A. Abdo et al. (Fermi/LAT Collaboration): Fermi Large Area Telescope First Source Catalog, ApJS 188 (2010) 405.

[6] A. A. Abdo et al. (Fermi/LAT Collaboration): Fermi Large Area Telescope observations of misaligned active galactic nuclei, ApJ 720 (2010) 912.

[7] S. Antón et al.: The colour of the narrow line Syl-blazar 0324+3410,A\&A 490 (2008) 583.

[8] T. G. Arshakian et al.: Radio-optical-gamma-ray properties of MOJAVE AGN detected by Fermi/LAT, A\&A, submitted [arXiv:1104.4946].

[9] W. B. Atwood et al. (Fermi/LAT Collaboration): The Large Area Telescope on the Fermi Gamma-Ray Space Telescope Mission, ApJ 697 (2009) 1071.

[10] J. N. Bahcall et al.: Hubble Space Telescope images of nearby luminous quasars. II. Results for eight quasars and tests of the detection sensitivity, ApJ $\mathbf{4 5 0}$ (1995) 486.

[11] R. H. Becker et al.: The FIRST Survey: Faint Images of the Radio Sky at Twenty Centimeters, ApJ 450 (1995) 559.

[12] R. D. Blandford \& M. J. Rees: Some comments on radiation mechanisms in Lacertids. In: Proceedings of the Pittsburgh Conference on BL Lac Objects, University of Pittsburgh (1978) p. 328. 
[13] R. D. Blandford \& A. Königl: Relativistic jets as compact radio sources, ApJ 232 (1979) 34.

[14] T. Boller et al.: Soft X-ray properties of narrow-line Seyfert 1 galaxies, A\&A 305 (1996) 53.

[15] M. Böttcher \& C. D. Dermer: An evolutionary scenario for blazar unification, ApJ 564 (2002) 86.

[16] T. A. Boroson \& R. F. Green: The emission-line properties of low-redshift quasi-stellar objects, ApJS 80 (1992) 109.

[17] T. A. Boroson: Black hole mass and Eddington ratio as drivers for the observable properties of radio-loud and radio-quiet QSOs, ApJ 565 (2002) 78.

[18] T. A. Boroson: NLS1 properties and demographics. In: Proceedings of the Workshop Narrow-Line Seyfert 1 Galaxies and Their Place in the Universe, PoS (NLS1) 003 (2011).

[19] A. Brunthaler et al.: III Zw 2, the first superluminal het in a Seyfert galaxy, A\&A 357 (2000) L45.

[20] A. Brunthaler et al.: The extreme flare in III Zw 2: evolution of a radio jet in a Seyfert galaxy, A\&A 435 (2005) 497.

[21] S. Buttiglione et al.: An optical spectroscopic survey of the 3CR sample of radio galaxies with $z<0.3$ . II. Spectroscopic classes and accretion modes in radio-loud AGN, A\&A $\mathbf{5 0 9}$ (2010) A6.

[22] J. A. Cardelli et al.: The relationship between infrared, optical, and ultraviolet extinction, ApJ 345 (1989) 245.

[23] A. Cavaliere \& V. D’Elia: The blazar main sequence, ApJ 571 (2002) 226.

[24] T. S. Chonis \& C. M. Gaskell: Setting UBVRI photometric zero-points using Sloan Digital Sky Survey ugriz magnitudes, AJ 135 (2008) 264.

[25] J. J. Condon et al.: The NRAO VLA Sky Survey, AJ 115 (1998) 1693.

[26] D. M. Crenshaw et al.: The host galaxies of narrow-line Seyfert 1 galaxies: evidence for bar-driven fueling, AJ 126 (2003) 1690.

[27] K. Davidson \& T. D. Kinman: On the possible importance of Markarian 359, ApJ 225 (1978) 776.

[28] R. Decarli et al.: Are the black hole masses in narrow-line Seyfert 1 galaxies actually small?, MNRAS 386 (2008) L15.

[29] R. P. Deo et al.: The host galaxies of narrow-line Seyfert 1 galaxies: nuclear dust morphology and starburst rings, AJ 132 (2006) 321.

[30] A. Doi et al.: VLBI observations of the most radio-loud narrow-line quasar SDSS J094857.3+002225, PASJ 58 (2006) 829.

[31] H. Ebeling et al.: ASMOOTH: a simple and efficient algorithm for adaptive kernel smoothing of two-dimensional imaging data, MNRAS 368 (2006) 65.

[32] B. H. C. Emonts et al.: Enormous disc of cool gas surrounding the nearby powerful radio galaxy NGC 612 (PKS 0131-36), MNRAS 387 (2008) 197.

[33] B. H. C. Emonts et al.: The disc-dominated host galaxy of FR-I radio source B2 0722+30, MNRAS 396 (2009) 1522.

[34] A. D. Falcone et al.: A search for TeV gamma-ray emission from high-peaked flat-spectrum radio quasars using the Whipple air Čerenkov telescope, ApJ 613 (2004) 710.

[35] E. .D Feigelson \& P. I. Nelson: Statistical methods for astronomical data with upper limits. I Univariate distributions, ApJ 293 (1985) 192. 
[36] S. Fine et al.: Orientation effects in quasar spectra: the broad-and narrow-line regions, MNRAS 412 (2011) 213.

[37] L. Foschini et al.: Blazar nuclei in radio-loud narrow-line Seyfert 1?, Adv. Space Res. 43 (2009) 889.

[38] L. Foschini et al.: Fermi/LAT discovery of gamma-ray emission from a relativistic jet in the narrow-line Seyfert 1 quasar PMN J0948+0022. In: Accretion and Ejection in AGNs: a global view, L. Maraschi et al. eds, ASP Conference Series 427 (2010) 243.

[39] L. Foschini et al.: The first gamma-ray outburst of a narrow-line Seyfert 1 galaxy: the case of PMN J0948+0022 in July 2010, MNRAS, in press [arXiv: 1010.4434 ].

[40] A. Franceschini et al.: Supermassive Black Holes in Early-Type Galaxies: Relationship with Radio Emission and Constraints on the Black Hole Mass Function, MNRAS 297 (1998) 817.

[41] L. C. Gallo et al.: The spectral energy distribution of PKS 2004-447: a compact steep-spectrum source and possible radio-loud narrow-line Seyfert 1 galaxy, MNRAS 370 (2006) 245.

[42] C. M. Gaskell: Reddening of the narrow-line regions of active galactic nuclei and the intrinsic Balmer decrement II, Astrophys. Lett. 24 (1984) 43.

[43] G. Ghisellini et al.: A theoretical unifying scheme for gamma-ray bright blazars, MNRAS 301 (1998) 451.

[44] G. Ghisellini et al.: Jet and accretion power in the most powerful Fermi blazars, MNRAS 399 (2009) 2041.

[45] G. Ghisellini et al.: General physical properties of bright Fermi blazars, MNRAS 402 (2010) 497.

[46] G. Ghisellini et al.: The transition between BL Lac objects and Flat Spectrum Radio Quasars, MNRAS, accepted for publication, [arXiv:1012.0308].

[47] M. Giroletti \& F. Panessa: The faintest Seyfert radio cores revealed by VLBI, ApJ 706 (2009) L260.

[48] M. Giroletti et al.: Global e-VLBI observations of the gamma-ray narrow line Seyfert 1 PMN J0948+0022, A\&A 528 (2011) L11.

[49] M. Gliozzi et al.: A Panchromatic View of PKS 0558-504: An Ideal Laboratory to Study the Disk-Jet Link, ApJ 717 (2010) 1243.

[50] D. Grupe: A complete sample of soft X-ray selected AGNs. II. Statistical analysis, AJ 127 (2004) 1799.

[51] D. Grupe: Statistical analysis of an AGN sample with simultaneous UV and X-ray observations with Swift. In: Proceedings of the Workshop Narrow-Line Seyfert 1 Galaxies and Their Place in the Universe, PoS (NLS1) 004 (2011).

[52] D. Grupe et al.: The enigmatic soft X-ray AGN RX J0134.2-4258, A\&A 356 (2000) 11.

[53] T. S. Hamilton et al.: The luminosity function of QSO host galaxies, ApJ 576 (2002) 61.

[54] T. S. Hamilton et al.: The fundamental plane of QSOs and the relationship between host and nucleus, ApJ 678 (2008) 22.

[55] L. C. Ho: Nuclear activity in nearby galaxies, Annu. Rev. Astron. Astrophys. 46 (2008) 475.

[56] L. C. Ho \& C. Y. Peng: Nuclear luminosities and radio loudness of Seyfert nuclei, ApJ 555 (2001) 650 .

[57] D. C. Homan et al.: Intrinsic brightness temperatures of AGN jets, ApJ 642 (2006) L115. 
[58] Y. Ikejiri et al.: Photopolarimetric Monitoring of Blazars in the Optical and Near-Infrared Bands with the Kanata Telescope: I. Correlations between Flux, Color, and Polarization, PASJ, accepted for publication, [arXiv:1105.0255].

[59] K. J. Inskip et al.: A near-IR study of the host galaxies of 2 Jy radio sources at $0.03 \lesssim z \lesssim 0.5-I$. The data, MNRAS 407 (2010) 1739.

[60] T. Isobe et al.: Statistical methods for astronomical data with upper limits. II - Correlation and regression, ApJ 306 (1986) 490.

[61] E. J. D. Jolley \& Z. Kuncic: Jet-enhanced accretion growth of supermassive black holes, MNRAS 386 (2008) 989.

[62] P. M. W. Kalberla et al.: The Leiden/Argentine/Bonn (LAB) Survey of Galactic HI. Final data release of the combined LDS and IAR surveys with improved stray-radiation corrections, A\&A $\mathbf{4 4 0}$ (2005) 775.

[63] S. Kirhakos et al.: The host galaxies of three radio-loud quasars: 3C 48, 3C 345, and B2 1425+267, ApJ 520 (1999) 67.

[64] E. Komatsu et al.: Seven-year Wilkinson Microwave Anisotropy Probe (WMAP) observations: cosmological interpretation, ApJS 192 (2011) 18.

[65] S. Komossa et al.: Radio-loud narrow-line type 1 quasars, AJ 132 (2006) 531.

[66] Y. Y. Kovalev et al.: The relation between AGN gamma-ray emission and parsec-scale radio jets, ApJ 696 (2009) L17.

[67] M. P. Lavalley et al.: ASURV, Pennsylvania State University. Report for the period Jan 1990 - Feb 1992., Bull. Am. Astron. Soc. 24 (1992) 839.

[68] A. Laor: On Black Hole Masses and Radio Loudness in Active Galactic Nuclei, ApJ 543 (2000) L111.

[69] M. J. Ledlow et al.: An unusual radio galaxy in Abell 428: a large, powerful FRI source in a disk-dominated host, ApJ 495 (1998) 227.

[70] M. J. Ledlow et al.: A large-scale jet and FRI radio source in a spiral galaxy: the host properties and external environment, ApJ 552 (2001) 120.

[71] J.-P. Lenain et al.: Seyfert 2 galaxies in the GeV band: jets and starburst, A\&A 524 (2010) A72.

[72] J. Loveday: The APM bright galaxy catalogue, MNRAS 278 (1996) 1025.

[73] L. Maraschi \& F. Tavecchio: The jet-disk connection and blazar unification, ApJ 593 (2003) 667.

[74] E. Massaro et al.: Roma-BZCAT: a multifrequency catalogue of blazars, A\&A 495 (2009) 691.

[75] S. Mathur: Host galaxies of NLS1s. In: Proceedings of the Workshop Narrow-Line Seyfert 1 Galaxies and Their Place in the Universe, PoS (NLS1) 035 (2011).

[76] S. Mathur et al.: Evolution of active galaxies: black-hole mass-bulge relations for narrow line objects, New Astron. 6, (2001) 321.

[77] S. Mathur et al.: Supermassive black holes, pseudobulges, and the narrow-line Seyfert 1 galaxies, ApJ, submitted [arXiv:1102.0537].

[78] J. R. Mattox et al.: The likelihood analysis of EGRET data, ApJ 461 (1996) 396.

[79] R. J. McLure \& J. S. Dunlop: On the black hole-bulge mass relation in active and inactive galaxies, MNRAS 331 (2002) 795. 
[80] M. Moles et al.: The nature of the N-galaxy 3C 120, A\&A 197 (1988) 1.

[81] D. G. Monet et al.: The USNO-B Catalog, AJ 125 (2003) 984.

[82] R. Ojha et al.: Photometric Observations of Selected, Optically Bright Quasars for Space Interferometry Mission and Other Future Celestial Reference Frames, AJ 138 (2009) 845.

[83] G. Orban de Xivry et al.: The role of secular evolution in the black hole growth of narrow-line Seyfert 1 galaxies, MNRAS, submitted [arXiv: 1104.5023$]$.

[84] A. Y. K. N. Oshlack et al.: A very radio loud narrow-line Seyfert 1: PKS 2004-447, ApJ 558 (2001) 578.

[85] D. E. Osterbrock \& R. W. Pogge: The spectra of narrow-line Seyfert 1 galaxies, ApJ 297 (1985) 166.

[86] E. S. Perlman et al.: The apparent host galaxy of PKS 1413+135: Hubble Space Telescope, ASCA, and Very Long Baseline Array observations, AJ 124 (2002) 2401.

[87] B. M. Peterson: Masses of Black Holes in Active Galactic Nuclei: Implications for Narrow-Line Seyfert 1 Galaxies. In: Proceedings of the Workshop Narrow-Line Seyfert 1 Galaxies and Their Place in the Universe, POS (NLS1) 032 (2011).

[88] B. M. Peterson et al.: X-ray and optical variability in NGC 4051 and the nature of narrow-line Seyfert 1 galaxies, ApJ 542, (2000), 161.

[89] R. W. Pogge: A quarter century of Narrow-Line Sefert 1s. In: Proceedings of the Workshop Narrow-Line Seyfert 1 Galaxies and Their Place in the Universe, POS (NLS1) 002 (2011).

[90] A. C. S. Readhead: Equipartition brightness temperature and the inverse Compton catastrophe, ApJ 426 (1994) 51.

[91] R. A. Remillard et al.: A rapid energetic X-ray flare in the quasar PKS 0558-504, Nature 350 (1986) 589.

[92] E. Sani et al.: Enhanced star formation in narrow-line Seyfert 1 active galactic nuclei revealed by Spitzer, MNRAS 403 (2010) 1246.

[93] M. Sikora et al.: Radio loudness of active galactic nuclei: observational facts and theoretical implications, ApJ 658 (2007) 815.

[94] M. Sikora et al.: Constraining Emission Models of Luminous Blazar Sources, ApJ 704 (2009) 38.

[95] C. M. Urry \& P. Padovani: Unified schemes for radio-loud active galactic nuclei, PASP 107 (1995) 803.

[96] S. van den Bergh: Galaxy morphology and classification, Cambridge University Press, Cambridge (1998).

[97] M. P. Véron-Cetty \& P. Véron: Are all radio galaxies genuine ellipticals?, A\&A 375 (2001) 791.

[98] W. Voges et al.: The ROSAT all-sky survey bright source catalogue, A\&A 349 (1999) 389.

[99] W. Voges et al.: ROSAT All-Sky Survey Faint Source Catalogue, IAU Circular 7432 (2000) 1.

[100] S. Wagner \& A. Witzel: Intraday variability in quasars and BL Lac objects, Annu. Rev. Astron. Astrophys. 33 (1995) 163.

[101] D. J. Whalen et al.: Optical properties of radio-selected narrow-line Seyfert 1 galaxies, AJ 131 (2006) 1948.

[102] B. J. Wills \& I. W. A. Browne: Relativistic beaming and quasar emission lines, ApJ 302 (1986) 56. 
[103] A. E. Wright et al.: The Parkes-MIT-NRAO (PMN) surveys. II. Source catalog for the southern survey $\left(-87.5^{\circ}<\delta<-37^{\circ}\right)$, ApJS 91 (1994) 111.

[104] W. Yuan et al.: A population of radio-loud narrow-line Seyfert 1 galaxies with blazar-like properties?, ApJ $\mathbf{6 8 5}$ (2008) 801.

[105] H.-Y. Zhou \& T.-G. Wang: Properties of broad band continuum of narrow-line Seyfert 1 galaxies, Chin. J. Astron. Astrophys. 2 (2002) 501.

[106] H.-Y. Zhou et al.: SDSS J094857.3+002225: a very radio loud narrow-line quasar with relativistic jets?, ApJ 584 (2003) 147.

[107] H.-Y. Zhou et al.: A narrow-line Seyfert 1-blazar composite nucleus in 2MASX J0324+3410, ApJ 658 (2007) L13. 


\section{A. List of NLS1}

The sample of 76 NLS1s is reported in the following Table A1. The MW data are those observed (Table A2), i.e. not corrected, except for $R$, which has been calculated after having dereddened the optical flux.

\begin{tabular}{|c|c|c|c|c|c|c|}
\hline Name & $\begin{array}{r}\text { RA } \\
\text { J2000 }\end{array}$ & $\begin{array}{r}\text { Dec } \\
\text { J2000 }\end{array}$ & $z$ & $\begin{array}{c}N_{\mathrm{H}} \\
{\left[10^{20} \mathrm{~cm}^{-2}\right]}\end{array}$ & $\begin{array}{r}\text { FWHM H } \beta \\
{[\mathrm{km} / \mathrm{s}]}\end{array}$ & $R$ \\
\hline FBQS J0022-1039 & 5.7051 & -10.6656 & 0.414 & 3.11 & 1845 & 6 \\
\hline FBQS J0100-0200 & 15.1342 & -2.0128 & 0.227 & 4.05 & 920 & 8 \\
\hline PMN J0134-4258 & 23.5704 & -42.9742 & 0.238 & 1.69 & 930 & 209 \\
\hline $1 \mathrm{H} 0323+342$ & 51.1715 & +34.1794 & 0.061 & 11.7 & 1600 & 318 \\
\hline PKS 0558-504 & 89.9474 & -50.4478 & 0.137 & 3.46 & 1500 & 27 \\
\hline FBQS J0706+3901 & 106.6048 & +39.0310 & 0.086 & 8.07 & 664 & 7 \\
\hline FBQS J0713+3820 & 108.4179 & +38.3444 & 0.123 & 6.00 & 1487 & 20 \\
\hline FBQS J0729+3046 & 112.4679 & +30.7792 & 0.150 & 6.00 & 891 & 2 \\
\hline FBQS J0736+3926 & 114.0964 & +39.4383 & 0.118 & 5.76 & 1806 & 3 \\
\hline FBQS J0744+5149 & 116.0096 & +51.8216 & 0.460 & 4.83 & 1989 & 59 \\
\hline FBQS J0752+2617 & 118.1900 & +26.2933 & 0.082 & 3.42 & 1906 & 2 \\
\hline FBQS J0758+3920 & 119.5002 & +39.3414 & 0.095 & 5.12 & 1908 & 90 \\
\hline FBQS J0804+3853 & 121.0385 & +38.8969 & 0.151 & 5.16 & 1356 & 10 \\
\hline RGB J0806+728 & 121.6624 & +72.8057 & 0.098 & 2.98 & 1060 & 41 \\
\hline FBQS J0810+2341 & 122.6086 & +23.6989 & 0.133 & 4.50 & 1831 & 6 \\
\hline RGB J0814+561 & 123.6338 & +56.1657 & 0.509 & 4.49 & 2164 & 361 \\
\hline FBQS J0818+3834 & 124.7053 & +38.5711 & 0.160 & 3.84 & 1683 & 6 \\
\hline SBS $0846+513$ & 132.4916 & +51.1414 & 0.584 & 3.00 & 1811 & 4496 \\
\hline SDSS J085001.17+462600.5 & 132.5049 & +46.4335 & 0.523 & 2.64 & 1251 & 318 \\
\hline PMN J0902+0442 & 135.6132 & +4.7193 & 0.532 & 3.11 & 2089 & 1974 \\
\hline FBQS J0909+3124 & 137.4494 & +31.4121 & 0.265 & 1.74 & 1610 & 8 \\
\hline FBQS J0937+3615 & 144.2626 & +36.2603 & 0.180 & 1.21 & 1048 & 12 \\
\hline FBQS J0946+3223 & 146.5458 & +32.3906 & 0.405 & 1.52 & 1615 & 5 \\
\hline PMN J0948+0022 & 147.2388 & +0.3738 & 0.585 & 5.20 & 1432 & 846 \\
\hline SDSS J095317.09+283601.5 & 148.3212 & +28.6004 & 0.657 & 1.28 & 2162 & 665 \\
\hline RBS 826 & 151.4244 & +43.5445 & 0.179 & 1.04 & 2059 & 4 \\
\hline FBQS J1010+3003 & 152.5029 & +30.0560 & 0.256 & 2.35 & 1305 & 2 \\
\hline SDSS J103123.73+423439.3 & 157.8489 & +42.5776 & 0.376 & 0.99 & 1642 & 291 \\
\hline SDSS J103727.45+003635.6 & 159.3644 & +0.6099 & 0.595 & 4.87 & 1357 & 569 \\
\hline FBQS J1038+4227 & 159.7483 & +42.4617 & 0.220 & 1.49 & 1979 & 10 \\
\hline B3 $1044+476$ & 161.8861 & +47.4256 & 0.798 & 1.30 & 2153 & 12281 \\
\hline RX J1048.3+2222 & 162.0691 & +22.3775 & 0.329 & 1.46 & 1301 & 10 \\
\hline FBQS J1102+2239 & 165.5974 & +22.6557 & 0.455 & 1.21 & 1972 & 32 \\
\hline $1107+372$ & 167.5210 & +36.8934 & 0.630 & 1.69 & 1300 & 1393 \\
\hline B2 $1111+32$ & 168.6621 & +32.6926 & 0.189 & 1.86 & 1980 & 1986 \\
\hline FBQS J1127+2654 & 171.9016 & +26.9140 & 0.379 & 1.36 & 1903 & 4 \\
\hline FBQS J1136+3432 & 174.2331 & +34.5436 & 0.193 & 1.71 & 918 & 4 \\
\hline SDSS J113824.54+365327.1 & 174.6023 & +36.8908 & 0.356 & 1.75 & 1364 & 328 \\
\hline SDSS J114654.28+323652.3 & 176.7262 & +32.6145 & 0.465 & 1.42 & 2081 & 154 \\
\hline FBQS J1159+2838 & 179.8222 & +28.6374 & 0.209 & 1.65 & 1415 & 20 \\
\hline FBQS J1220+3853 & 185.1463 & +38.8879 & 0.377 & 2.21 & 1917 & 4 \\
\hline FBQS J1227+3214 & 186.9548 & +32.2497 & 0.137 & 1.62 & 951 & 91 \\
\hline SDSS J123852.12+394227.8 & 189.7173 & +39.7077 & 0.622 & 1.46 & 910 & 268 \\
\hline SDSS J124634.65+023809.0 & 191.6444 & +2.6358 & 0.362 & 2.01 & 1425 & 277 \\
\hline FBQS J1256+3852 & 194.0086 & +38.8752 & 0.419 & 1.73 & 2079 & 8 \\
\hline SDSS J130522.75+511640.3 & 196.3448 & +51.2778 & 0.785 & 0.98 & 1925 & 277 \\
\hline FBQS J1333+4141 & 203.4395 & +41.6910 & 0.225 & 0.74 & 1940 & 9 \\
\hline FBQS J1346+3121 & 206.6457 & +31.3594 & 0.246 & 1.27 & 1600 & 11 \\
\hline
\end{tabular}

Table A1: Sample of NLS1s. 


\begin{tabular}{lrrccrr}
\hline Name & RA & Dec & $z$ & $N_{\mathrm{H}}$ & FWHM H $\beta$ & $R$ \\
\hline FBQS J1358+2658 & 209.6891 & +26.9690 & 0.331 & 1.55 & 1863 & 11 \\
FBQS J1405+2555 & 211.3176 & +25.9261 & 0.165 & 1.29 & 1398 & 1 \\
FBQS J1408+2409 & 212.1159 & +24.1569 & 0.131 & 1.57 & 1590 & 4 \\
FBQS J1421+2824 & 215.3086 & +28.4145 & 0.540 & 1.28 & 1838 & 204 \\
SDSS J143509.49+313147.8 & 218.7897 & +31.5301 & 0.501 & 1.14 & 1719 & 949 \\
FBQS J1442+2623 & 220.6700 & +26.3924 & 0.108 & 2.13 & 795 & 5 \\
B3 1441+476 & 220.8273 & +47.4324 & 0.703 & 1.47 & 1848 & 1067 \\
FBQS J1448+3559 & 222.1046 & +35.9963 & 0.114 & 1.00 & 1856 & 2 \\
PKS 1502+036 & 226.2770 & +3.4419 & 0.408 & 3.89 & 1082 & 3364 \\
FBQS J1517+2239 & 229.3844 & +22.6564 & 0.109 & 3.73 & 1789 & 6 \\
FBQS J1519+2838 & 229.9006 & +28.6410 & 0.270 & 2.28 & 1641 & 4 \\
RGB J1548+351 & 237.0747 & +35.1912 & 0.478 & 2.28 & 2035 & 701 \\
FBQS J1612+4219 & 243.2493 & +42.3279 & 0.233 & 1.19 & 819 & 24 \\
RGB J1629+401 & 247.2555 & +40.1332 & 0.272 & 1.05 & 1260 & 50 \\
RGB J1633+473 & 248.3483 & +47.3164 & 0.116 & 1.79 & 909 & 154 \\
SDSS J163401.94+480940.2 & 248.5081 & +48.1612 & 0.494 & 1.64 & 1609 & 187 \\
RGB J1644+263 & 251.1772 & +26.3203 & 0.144 & 5.12 & 1507 & 396 \\
FBQS J1702+3247 & 255.6294 & +32.7888 & 0.164 & 1.71 & 1400 & 1 \\
B3 1702+457 & 255.8766 & +45.6798 & 0.060 & 2.52 & 490 & 102 \\
FBQS J1713+3523 & 258.2686 & +35.3926 & 0.085 & 2.45 & 1002 & 10 \\
FBQS J1716+3112 & 259.0081 & +31.2038 & 0.110 & 3.00 & 1571 & 1 \\
FBQS J1718+3042 & 259.7096 & +30.7004 & 0.281 & 3.06 & 1434 & 3 \\
SDSS J172206.03+565451.6 & 260.5251 & +56.9143 & 0.425 & 2.10 & 1385 & 323 \\
PKS 2004-447 & 301.9799 & -44.5790 & 0.240 & 2.96 & 1447 & 6358 \\
PHL 1811 & 328.7563 & -9.3734 & 0.192 & 4.04 & 1500 & 1 \\
RX J2159.4+0113 & 329.8502 & +1.2182 & 0.101 & 4.27 & 1429 & 3 \\
FBQS J2327-1023 & 351.8105 & -10.3882 & 0.065 & 2.25 & 652 & 1 \\
FBQS J2338-0900 & 354.6415 & -9.0109 & 0.374 & 2.21 & 1564 & 9 \\
\hline & & & & & &
\end{tabular}

Table A1: - Continue. 


\begin{tabular}{|c|c|c|c|c|}
\hline Name & $\begin{array}{l}f_{1.4 \mathrm{GHz}} \\
{[\mathrm{mJy}]}\end{array}$ & $\begin{array}{c}m_{B} \\
{[\mathrm{mag}]}\end{array}$ & $\begin{array}{c}F_{0.1-2.4 \mathrm{keV}} \\
{\left[10^{-12} \mathrm{erg} \mathrm{cm}^{-2} \mathrm{~s}^{-1}\right]}\end{array}$ & $\begin{array}{c}F_{0.1-100 \mathrm{GeV}} \\
{\left[10^{-8} \mathrm{ph} \mathrm{cm}^{-2} \mathrm{~s}^{-1}\right]}\end{array}$ \\
\hline FBQS J0022-1039 & $1.9 \pm 0.1$ & $17.81 \pm 0.05$ & $<0.2$ & $<0.15$ \\
\hline FBQS J0100-0200 & $5.9 \pm 0.1$ & $17.1 \pm 0.1$ & $<0.2$ & $<0.11$ \\
\hline PMN J0134-4258 & $55 \pm 9$ & $18.0 \pm 0.3$ & $2.4 \pm 0.3$ & $<2.6$ \\
\hline $1 \mathrm{H} 0323+342$ & $613 \pm 21$ & $16.38 \pm 0.03$ & $8.0 \pm 0.7$ & $6.0 \pm 0.7$ \\
\hline PKS 0558-504 & $121 \pm 10$ & $15.08 \pm 0.01$ & $88 \pm 6$ & $<2.4$ \\
\hline FBQS J0706+3901 & $4.3 \pm 0.5$ & $17.6 \pm 0.3$ & $<0.3$ & $<0.15$ \\
\hline FBQS J0713+3820 & $10.8 \pm 0.1$ & $15.1 \pm 0.3$ & $3.2 \pm 0.5$ & $<3.1$ \\
\hline FBQS J0729+3046 & $1.2 \pm 0.1$ & $17.4 \pm 0.3$ & $0.50 \pm 0.22$ & $<2.1$ \\
\hline FBQS J0736+3926 & $3.6 \pm 0.1$ & $16.53 \pm 0.06$ & $9.0 \pm 0.8$ & $<0.16$ \\
\hline FBQS J0744+5149 & $11.9 \pm 0.1$ & $18.4 \pm 0.3$ & $1.2 \pm 0.3$ & $<0.084$ \\
\hline FBQS J0752+2617 & $1.3 \pm 0.1$ & $17.06 \pm 0.05$ & $3.3 \pm 0.4$ & $<2.0$ \\
\hline FBQS J0758+3920 & $11.6 \pm 0.1$ & $19.08 \pm 0.08$ & $2.0 \pm 0.5$ & $<3.1$ \\
\hline FBQS J0804+3853 & $2.7 \pm 0.1$ & $18.27 \pm 0.08$ & $<0.3$ & $<0.066$ \\
\hline RGB J0806+728 & $50.1 \pm 0.2$ & $16.5 \pm 0.3$ & $2.9 \pm 0.6$ & $<1.7$ \\
\hline FBQS J0810+2341 & $0.9 \pm 0.1$ & $18.89 \pm 0.07$ & $0.99 \pm 0.35$ & $<4.2$ \\
\hline RGB J0814+561 & $80.2 \pm 0.2$ & $18.27 \pm 0.04$ & $1.2 \pm 0.2$ & $<1.4$ \\
\hline FBQS J0818+3834 & $2.1 \pm 0.1$ & $17.92 \pm 0.06$ & $<0.2$ & $<3.7$ \\
\hline SBS $0846+513$ & $350.0 \pm 0.1$ & $19.27 \pm 0.07$ & $0.23 \pm 0.03$ & $0.51 \pm 0.15$ \\
\hline SDSS J085001.17+462600.5 & $21.3 \pm 0.1$ & $19.43 \pm 0.06$ & $<0.18$ & $<3.4$ \\
\hline PMN J0902+0442 & $156.5 \pm 0.2$ & $19.28 \pm 0.06$ & $<0.2$ & $<2.7$ \\
\hline FBQS J0909+3124 & $1.5 \pm 0.1$ & $18.35 \pm 0.08$ & $<0.14$ & $<1.3$ \\
\hline FBQS J0937+3615 & $3.2 \pm 0.1$ & $17.99 \pm 0.07$ & $1.2 \pm 0.2$ & $<2.1$ \\
\hline FBQS J0946+3223 & $1.6 \pm 0.1$ & $17.81 \pm 0.05$ & $<0.13$ & $<7.0$ \\
\hline PMN J0948+0022 & $111.5 \pm 0.1$ & $18.86 \pm 0.05$ & $1.0 \pm 0.3$ & $13.7 \pm 0.7$ \\
\hline SDSS J095317.09+283601.5 & $47.9 \pm 0.2$ & $19.21 \pm 0.04$ & $<0.12$ & $<1.4$ \\
\hline RBS 826 & $2.8 \pm 0.1$ & $16.87 \pm 0.05$ & $7.4 \pm 0.5$ & $<0.39$ \\
\hline FBQS J1010+3003 & $1.0 \pm 0.1$ & $17.23 \pm 0.05$ & $2.2 \pm 0.3$ & $<6.5$ \\
\hline SDSS J103123.73+423439.3 & $17.0 \pm 0.1$ & $19.52 \pm 0.07$ & $<0.1$ & $<3.1$ \\
\hline SDSS J103727.45+003635.6 & $27.9 \pm 0.1$ & $19.91 \pm 0.06$ & $<0.25$ & $<2.7$ \\
\hline FBQS J1038+4227 & $2.4 \pm 0.1$ & $18.11 \pm 0.07$ & $<0.13$ & $<0.071$ \\
\hline B3 $1044+476$ & $789 \pm 24$ & $19.29 \pm 0.06$ & $0.27 \pm 0.11$ & $<0.78$ \\
\hline RX J1048.3+2222 & $1.5 \pm 0.1$ & $18.62 \pm 0.06$ & $1.3 \pm 0.3$ & $<0.38$ \\
\hline FBQS J1102+2239 & $1.8 \pm 0.1$ & $19.55 \pm 0.07$ & $0.62 \pm 0.26$ & $2.0 \pm 0.6$ \\
\hline $1107+372$ & $23.3 \pm 0.8$ & $20.83 \pm 0.08$ & $<0.14$ & $<0.73$ \\
\hline B2 $1111+32$ & $107.8 \pm 0.1$ & $19.7 \pm 0.1$ & $<0.15$ & $<0.62$ \\
\hline FBQS J1127+2654 & $1.9 \pm 0.1$ & $17.24 \pm 0.06$ & $<0.13$ & $<3.1$ \\
\hline FBQS J1136+3432 & $1.3 \pm 0.1$ & $17.88 \pm 0.06$ & $<0.14$ & $<0.11$ \\
\hline SDSS J113824.54+365327.1 & $12.6 \pm 0.1$ & $20.04 \pm 0.08$ & $<0.15$ & $<4.9$ \\
\hline SDSS J114654.28+323652.3 & $15.4 \pm 0.1$ & $18.93 \pm 0.05$ & $0.22 \pm 0.10$ & $<5.1$ \\
\hline FBQS J1159+2838 & $2.0 \pm 0.1$ & $19.04 \pm 0.07$ & $<0.14$ & $<2.1$ \\
\hline FBQS J1220+3853 & $2.2 \pm 0.1$ & $17.14 \pm 0.05$ & $0.41 \pm 0.16$ & $<1.5$ \\
\hline FBQS J1227+3214 & $6.4 \pm 0.1$ & $19.5 \pm 0.1$ & $0.98 \pm 0.28$ & $<0.41$ \\
\hline SDSS J123852.12+394227.8 & $11.2 \pm 0.1$ & $19.84 \pm 0.05$ & $0.66 \pm 0.13$ & $<0.98$ \\
\hline SDSS J124634.65+023809.0 & $38.1 \pm 0.1$ & $18.67 \pm 0.05$ & $<0.16$ & $1.7 \pm 0.7$ \\
\hline FBQS J1256+3852 & $2.1 \pm 0.1$ & $17.90 \pm 0.05$ & $<0.14$ & $<0.15$ \\
\hline SDSS J130522.75+511640.3 & $86.9 \pm 0.1$ & $17.54 \pm 0.05$ & $<0.11$ & $<1.6$ \\
\hline FBQS J1333+4141 & $2.0 \pm 0.1$ & $18.10 \pm 0.06$ & $0.49 \pm 0.10$ & $<2.9$ \\
\hline FBQS J1346+3121 & $1.4 \pm 0.1$ & $18.77 \pm 0.06$ & $<0.12$ & $<0.087$ \\
\hline FBQS J1358+2658 & $1.2 \pm 0.1$ & $18.95 \pm 0.07$ & $<0.14$ & $<2.0$ \\
\hline
\end{tabular}

Table A2: Sample of NLS1s: observed data (i.e. not corrected). 


\begin{tabular}{|c|c|c|c|c|}
\hline Name & $\begin{array}{l}f_{1.4 \mathrm{GHz}} \\
{[\mathrm{mJy}]}\end{array}$ & $\begin{array}{c}m_{B} \\
{[\mathrm{mag}]}\end{array}$ & $\begin{array}{c}F_{0.1-2.4 \mathrm{keV}} \\
{\left[10^{-12} \mathrm{erg} \mathrm{cm}^{-2} \mathrm{~s}^{-1}\right]}\end{array}$ & $\begin{array}{c}F_{0.1-100 \mathrm{GeV}} \\
{\left[10^{-8} \mathrm{ph} \mathrm{cm}^{-2} \mathrm{~s}^{-1}\right]}\end{array}$ \\
\hline FBQS J1405+2555 & $0.7 \pm 0.1$ & $15.46 \pm 0.04$ & $8.1 \pm 0.5$ & $<0.047$ \\
\hline FBQS J1408+2409 & $3.0 \pm 0.1$ & $16.96 \pm 0.06$ & $3.4 \pm 0.4$ & $<0.64$ \\
\hline FBQS J1421+2824 & $48.7 \pm 0.1$ & $17.95 \pm 0.04$ & $<0.13$ & $<0.078$ \\
\hline SDSS J143509.49+313147.8 & $44.7 \pm 0.1$ & $19.72 \pm 0.06$ & $1.2 \pm 0.2$ & $<1.1$ \\
\hline FBQS J1442+2623 & $3.4 \pm 0.1$ & $17.06 \pm 0.07$ & $1.2 \pm 0.2$ & $<1.0$ \\
\hline B3 $1441+476$ & $171.1 \pm 0.1$ & $18.35 \pm 0.04$ & $0.29 \pm 0.12$ & $<1.1$ \\
\hline FBQS J1448+3559 & $1.5 \pm 0.1$ & $16.87 \pm 0.06$ & $4.2 \pm 0.3$ & $<0.14$ \\
\hline PKS $1502+036$ & $380.5 \pm 0.1$ & $18.99 \pm 0.06$ & $<0.22$ & $7.0 \pm 0.6$ \\
\hline FBQS J1517+2239 & $1.1 \pm 0.1$ & $18.56 \pm 0.07$ & $0.43 \pm 0.19$ & $<1.0$ \\
\hline FBQS J1519+2838 & $2.0 \pm 0.1$ & $17.34 \pm 0.06$ & $0.65 \pm 0.21$ & $<1.9$ \\
\hline RGB J1548+351 & $141.5 \pm 0.1$ & $18.22 \pm 0.05$ & $0.92 \pm 0.31$ & $<1.4$ \\
\hline FBQS J1612+4219 & $3.6 \pm 0.1$ & $18.56 \pm 0.06$ & $<0.12$ & $<0.14$ \\
\hline RGB J1629+401 & $11.9 \pm 0.2$ & $18.04 \pm 0.05$ & $8.8 \pm 0.3$ & $<1.2$ \\
\hline RGB J1633+473 & $65.0 \pm 0.1$ & $17.55 \pm 0.06$ & $2.8 \pm 0.3$ & $<0.097$ \\
\hline SDSS J163401.94+480940.2 & $7.7 \pm 0.1$ & $19.89 \pm 0.06$ & $0.20 \pm 0.08$ & $<1.0$ \\
\hline RGB J1644+263 & $90.8 \pm 0.2$ & $18.40 \pm 0.06$ & $2.8 \pm 0.5$ & $<1.9$ \\
\hline FBQS J1702+3247 & $1.5 \pm 0.1$ & $16.15 \pm 0.05$ & $8.1 \pm 0.4$ & $<2.8$ \\
\hline B3 $1702+457$ & $118.6 \pm 0.1$ & $16.52 \pm 0.08$ & $16.1 \pm 0.7$ & $<1.3$ \\
\hline FBQS J1713+3523 & $11.2 \pm 0.1$ & $16.56 \pm 0.06$ & $17.2 \pm 0.7$ & $<0.45$ \\
\hline FBQS J1716+3112 & $2.4 \pm 0.1$ & $16.18 \pm 0.05$ & $5.4 \pm 0.4$ & $<1.8$ \\
\hline FBQS J1718+3042 & $0.6 \pm 0.1$ & $18.45 \pm 0.06$ & $0.70 \pm 0.17$ & $<0.68$ \\
\hline SDSS J172206.03+565451.6 & $39.8 \pm 0.1$ & $18.76 \pm 0.06$ & $1.3 \pm 0.2$ & $<1.3$ \\
\hline PKS 2004-447 & $791 \pm 38$ & $18.9 \pm 0.3$ & $0.44 \pm 0.22$ & $1.2 \pm 0.3$ \\
\hline PHL 1811 & $1.2 \pm 0.1$ & $13.9 \pm 0.3$ & $<0.22$ & $<2.1$ \\
\hline RX J2159.4+0113 & $2.1 \pm 0.2$ & $17.28 \pm 0.06$ & $1.4 \pm 0.5$ & $<5.7$ \\
\hline FBQS J2327-1023 & $2.2 \pm 0.1$ & $16.02 \pm 0.07$ & $<0.17$ & $<2.2$ \\
\hline FBQS J2338-0900 & $1.8 \pm 0.1$ & $18.29 \pm 0.05$ & $<0.16$ & $<4.7$ \\
\hline
\end{tabular}

Table A2: - Continue. 